\title{
Caracterización fitoclimática de un sector de la montaña central cantábrica: el macizo de Curavacas (Palencia)
}

\section{Phytoclimatic characterization of a sector in cantabrian central mountain: Curavacas Massif (Palencia)}

\author{
Fernando Allende Álvarez, Nieves López Estébanez \\ y Felipe Fernández García ${ }^{1}$
}

\section{INTRODUCCIÓN}

En este trabajo se realiza una aproximación a la caracterización fitoclimática de un conjunto montañoso incluido en el sector palentino de las montañas cantábricas centrales y cuya culminación principal es el pico Curavacas (2.524 m, figura 1). En su mayor parte se asienta sobre una estructura sinclinoria dominada por conglomerados primarios y perteneciente a la región geológica del Pisuerga-Carrión (Rodríguez Fernández, 1994). La rigidez de los conglomerados westfalienses posibilita la existencia de un denso entramado de fracturación que determina una nítida compartimentación del macizo. Siguiendo las direcciones de las principales líneas de fractura el macizo se articula, especialmente en su vertiente septentrional, en diferentes umbrales y cubetas sobre los que ejerció su labor abrasiva el hielo pleistoceno (Frochoso $\&$ Castañón, 1996; Pellitero Ondicol, 2013a y 2013b).

A la singularidad de las geoformas heredadas y las litologías que lo conforman se suma la peculiaridad de sus comunidades vegetales. Es un sector de

\footnotetext{
${ }^{1}$ Departamento de Geografía, Universidad Autónoma de Madrid. fernando.allende@uam.es nieves.lopez@uam.es felipe.fernandez@uam.es
} 
transición entre el mundo atlántico y el mediterráneo en el que se mezclan taxones propios del centro y norte de Europa con otros característicos de la Submeseta Norte, típicamente mediterráneos. Es en estas altas montaña silíceas cantábricas donde aún pueden encontrarse algunas de las especies que florísticamente son nexos de unión con el pretérito continuum cántabro-pirenaico. El carácter de transición de estas montañas se acentúa en sus características climáticas, a medio camino entre los climas oceánicos de montaña y los submediterráneos según la clasificación propuesta por Martín Vide y Olcina Cantos (2001) y, en todo caso, incluidas en las montañas húmedas del oriente castellano-leonés (García Fernández, 1986).

FIGURA 1

LOCALIZACIÓN DEL ÁREA DE ESTUDIO

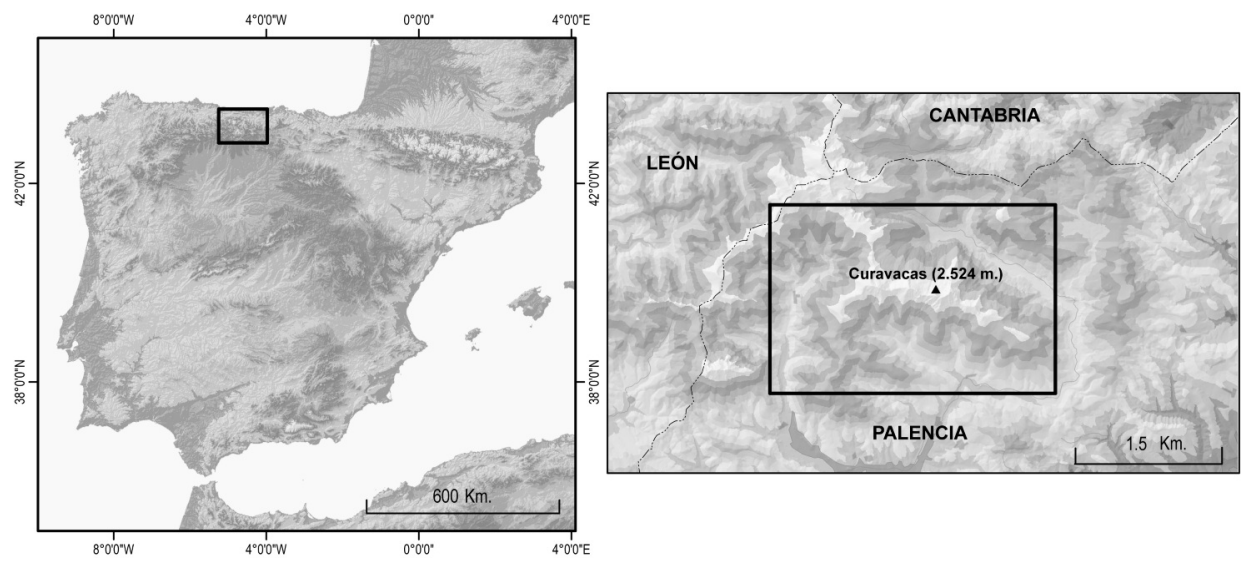

Fuente: elaboración propia a partir de MDE modificado de USGS Shuttle Radar Topography, 2004.

El área elegida está delimitada, al Norte, por el amplio valle de Pineda drenado por el río Carrión; al Este por el cordal que desciende desde El Hospital y el vallejo de Hoyo Muerto; al Oeste por las suaves vertientes en el entorno del Pozo del Vés; y, al Sur, por los afloramientos pizarrosos de El Resollar. Cuatro fueron los sectores muestreados en detalle: la vertiente de solana de El Resollar-Callejón Grande, el cordal del collado del Hospital, los pedregales y roquedos de la culminación-Aguja del Pastel y la vertiente septentrional, entre el Pozo Curavacas y los roquedales de cumbre (figura 2). 
FIGURA 2

LOCALIZACIÓN DETRANSECTOS E INVENTARIOS Y DETALLE DE LA CUMBRE

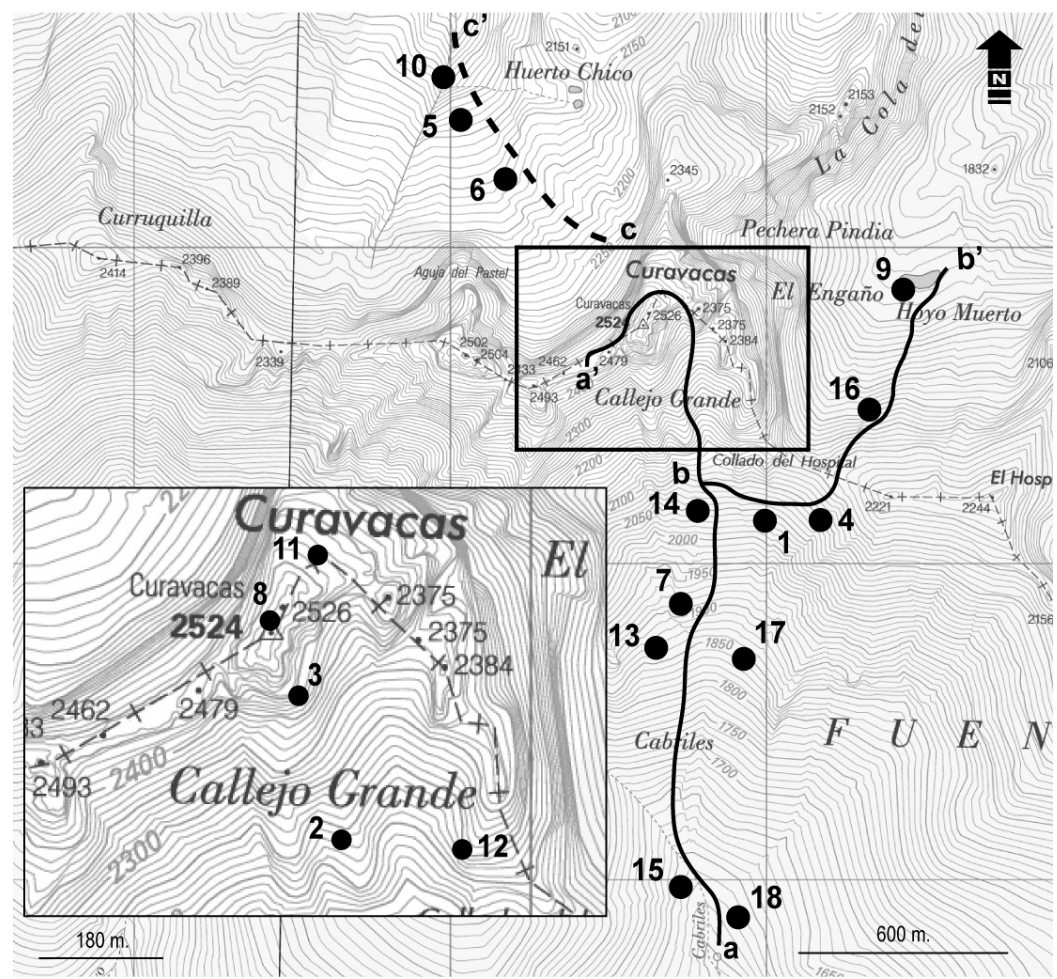

Fuente: elaboración propia a partir de la planimetría MTN25, IGN.

\section{MATERIAL Y MÉTODOS}

El trabajo se estructuró en cuatro fases: muestreo de campo, fotointerpretación de comunidades vegetales, elección y discriminación de variables y tratamiento estadístico e integración en un sistema de información geográfica.

En una primera fase se realizaron muestreos de campo en dos temporadas 2005-2006 (a-a', vid. figura 2) y 2007-2008 (b-b'/c-c', vid figura 2) y siempre en la segunda quincena del mes de julio. Previamente se consultaron diferentes fuentes asociadas a la flora orófila picoeuropeana (Rivas Martínez et al., 1984; Nava, 1988) y a los macizos conglomeráticos y metamórficos próximos (Herrero, 1986; Loriente Escallada, 1991; SIVIM, 2011). Los sectores a muestrear se 
eligieron mediante transectos previos que permitieron catalogar aquellas asociaciones de especies características, optimizando el posterior esfuerzo muestral. Las asociaciones se delimitaron mediante fotointerpretación sobre las imágenes del Plan Nacional de Ortofotografía Aérea (IGN. http://www.ign.es/descarga, enero 2012. Verificada http://www.ign.es/descarga). En cada muestreo se recogieron datos de geoposicionamiento con un GPS Trimble Nomad realizándose 18 inventarios que consideran métodos mixtos que contemplan la ausencia/presencia de las especies y el porcentaje cubierto por cada una de ellas². La elección de la superficie de muestreo varió en función de las características del terreno inventariado, desde las comunidades de menor superficie como los herbazales rupícolas umbro-acidófilos (aprox. $5 \mathrm{~m}^{2}$ ) a las que ocupan una mayor extensión como genistedos, piornales o lastonares (aprox. $20 \mathrm{~m}^{2}$ ). Se registraron además datos como la altitud y tipo de formación geomorfológica. En campo también se realizó una estimación cualitativa del porcentaje de cabida cubierta de cada especie diferenciando hasta cinco niveles fitoestructurales ${ }^{3}$.

La segunda fase se orientó a la modelización de las variables ${ }^{4}$. En ésta y, debido a la inexistencia de datos climáticos, se llevó a cabo la modelización de los mismos mediante la utilización de sistemas de información geográfica. En el caso de las variables climáticas se procesaron registros térmicos, pluviométricos y de espesor nival procedentes del Servicio de Información Geográfica de Datos Agrarios (series normales del INM) y del programa EHRIN (MOPTMA. 1994a, 1994b, 1995a, 1995b). Para los datos térmicos y pluviométricos se utilizaron las normales climáticas de 39 y 38 estaciones, datos obtenidos del SIGA (Servicio de Información Geográfica de Datos Agrarios) y con buena representatividad

\footnotetext{
${ }^{2}$ Conforme a los criterios establecidos por Braun Blanquet en cuanto a porcentajes y representatividad de taxones (1979) y, en cuanto a la elección de tamaño muestral y localización de los muestreos, algunas de las indicaciones recogidas en los documentos de trabajo del proyecto GLORIA (Pauli et al., 2003).

${ }^{3}$ Considerando su porte y biotipo diferenciamos: arbóreo, arborescente, arbustivo, subarbustivo y herbáceo.

${ }^{4}$ MDE-LIDAR, Instituto Geográfico Nacional; Datos termopluviométricos: Instituto Nacional de Meteorología estandarizados y disponibles en http://sig.marm.es/siga/; datos nivales: MOPTMA. Programa EHRIN. La nieve en las cordilleras españolas. Temporadas 91-92, 92-93, 93-94, 94-95. En la pluviometría se consideraron los valores medios anuales máximos y mínimos y valores mensuales medios máximos y mínimos. Para la termometría se consideraron las medias mensuales, medias mensuales del mes más cálido, medias mensuales del mes más frío, mínimas absolutas del mes más frío y las mínimas absolutas del mes más cálido. En el caso de la información más relacionada con la vegetación se calcularon índices como el de termicidad de Rivas Martínez et al. (1984), evapotranspiración potencial según Thornthwaite. Se incluye también la estimación de la insolación calculada en número de horas.
} 
espacial en ambas vertientes de la cordillera Cantábrica. También se utilizaron los datos de espesor nival disponibles en el programa EHRIN, bien repartidos espacialmente aunque con observaciones limitadas temporalmente. A partir de los datos anteriores se calcularon índices bioclimáticos como el de termicidad de Rivas Martínez. También se obtuvieron variables topofisiográficas calculadas de manera directa o indirecta como altitud, latitud, longitud, orientación, pendiente o distancia a la divisoria de aguas y al mar. La referencia de partida fue la obtenida a partir del Modelo Digital del Terreno LIDAR. Este modelo se adecua a los criterios y características en la creación y explotación del MDT (Hutchinson, 1993; Felicísimo Pérez, 1994) exigidos para nuestro trabajo.

En la tercera fase y, al igual que en trabajos previos (Allende, 2006; 2007; 2008), se obtuvieron variables de carácter continuo y de tipo ordinal. Resultó fundamental revisar metodologías mixtas para comprender y conseguir identificar las relaciones entre taxones y variables abióticas. Por ello se revisaron métodos previos orientados al análisis de variables climáticas (Fernández García, 1981; Fernández García et al., 1996) y fitoclimáticas (Felicísimo Pérez, 2011; Allende, 2006; Rivas Martínez, 2008; y también se profundizó en aquellas técnicas de carácter esencialmente multivariado que contrastan la validez (Bedia et al., 2011) en la aplicación al objetivo concreto de nuestra investigación (Tuhkanen, 1980; Podani, 1984; Escudero et al., 1984). Como variables independientes se seleccionaron aquellas que tuvieron una mayor influencia sobre la variabilidad espacial del clima, como la altitud, distancia al mar, distancia a la divisoria, latitud, longitud, altitud, orientación y pendiente. Los cálculos resultantes ${ }^{5}$ permitieron la obtención de una serie de ecuaciones para cada variable considerada y su representación a través de una estructura de datos tipo ESRI Grid floating-point. Tan sólo cuando los coeficientes de determinación fueron muy bajos, por ejemplo las precipitaciones, se optó por la reclasificación y agrupamiento de los observatorios (cuadro 1).

\footnotetext{
${ }^{5}$ En los cálculos aplicados a las variables climáticas se utilizan técnicas de análisis discriminante en el que se consideran como independientes un total de 7 (altura, latitud, longitud, orientación, pendiente, distancia al mar y distancia a la divisoria de aguas) y 4 dependientes (temperatura, espesor nival, pluviometría, evapotranspiración potencial y el índice de termicidad de Rivas Martínez). Los resultados permitieron modelizar espacialmente y mediante la obtención de ecuaciones la insolación, el espesor nival, pluviometría media, temperaturas, ETP y pisos bioclimáticos. Aplicadas las ecuaciones se realizó la extracción de la información para cada parcela de muestreo. Esto permitió obtener datos para cada una de las especies en las diferentes variables. El resultado fue una ingente cantidad de datos que, para su procesado y agrupamiento, fue necesario unificar mediante el método de las distancias euclídeas. Se pueden consultar para ampliar esta información trabajos previos en Allende (2006 y 2008).
} 
CUAdro 1

RESUMEN DE VARIABLES UTILIZADAS EN LA MODELIZACIÓN

DE DATOS CLIMÁTICOS Y FITOCLIMÁTICOS Y COEFICIENTES

DE DETERMINACIÓN OBTENIDOS

\begin{tabular}{|c|c|c|c|c|c|c|c|c|}
\hline & A & Dd & $\mathrm{Dm}$ & $\mathrm{La}$ & Lo & 0 & $\mathrm{P}$ & $\mathrm{r} 2$ \\
\hline Precipitación media mensual & $*$ & * & * & * & * & * & * & 0.75 \\
\hline Temperatura media mensual & * & & & * & * & * & & 0,91 \\
\hline Temperatura mínima absoluta anuales & * & & & * & * & * & & 0.86 \\
\hline Espesor nival & $*$ & & & & & & & 0.76 \\
\hline ETP mensual media anual Thornthwaite & * & * & * & * & * & * & * & 0.88 \\
\hline Indice Termicidad Rivas Martínez & * & * & * & * & * & * & * & 0.84 \\
\hline
\end{tabular}

* Variables independientes que intervienen en el cálculo

A: altura en metros; Dd, distancia a la divisoria; Dm, distancia al mar; La, latitud; Lo, longitud; O, orientación; P, pendiente (Fuente: elaboración propia a partir de Allende 2007 y 2008).

Por último, en la cuarta fase, se procedió a la plasmación gráfica de las diferentes variables consideradas en el análisis multivariado con el programa Arcgis 10.1. y considerando metodologías de análisis espacial como las aplicadas por Green y Cousins (1993), Fotheringham y Rogerson (1994) o Price y Heywood (1994).

\section{RESULTADOS}

Se consideran en primer lugar los datos climáticos obtenidos de la modelización entre los que se incluyen pluviometría, espesor de nieve, termometría e insolación e índices bioclimáticos (cuadro 2), así como la distribución espacial de algunos de ellos (figura 3). A continuación se caracterizan aquellas comunidades consideradas más significativas a excepción de los brezales de Erica australis y Calluna vulgaris que, aunque cartografiados, no han sido muestreados para este trabajo. Por último se abordan las relaciones clima-vegetación desde los datos proporcionados por los muestreos de campo y la modelización de los datos climáticos. 


\section{FIGURA 3}

MODELIZACIÓN DE LAS PRECIPITACIONES MEDIAS ANUALES (1), TEMPERATURAS MEDIAS MÍNIMAS ABSOLUTAS ANUALES (2), PISOS BIOCLIMÁTICOS (3) Y TEMPERATURAS MEDIAS MENSUALES ANUALES (4)

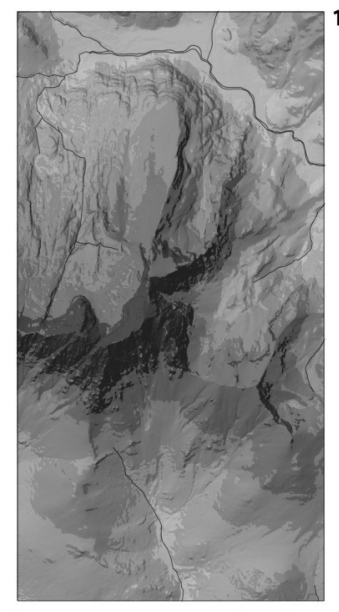

$1.176 \quad 1.517 \quad 1.745 \quad 1.953 \quad 2883 \mathrm{~mm}$.

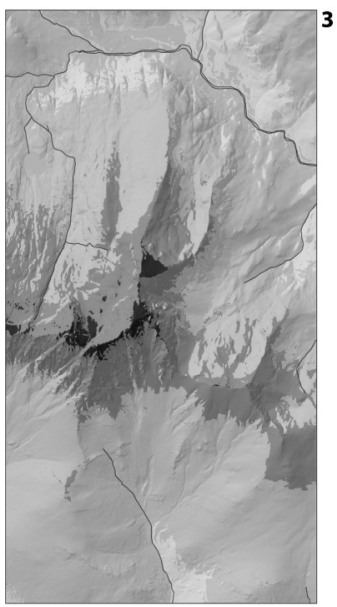

Altimontano Subalpino sup. $\square$ Subalpino inf. - Alpino inf.
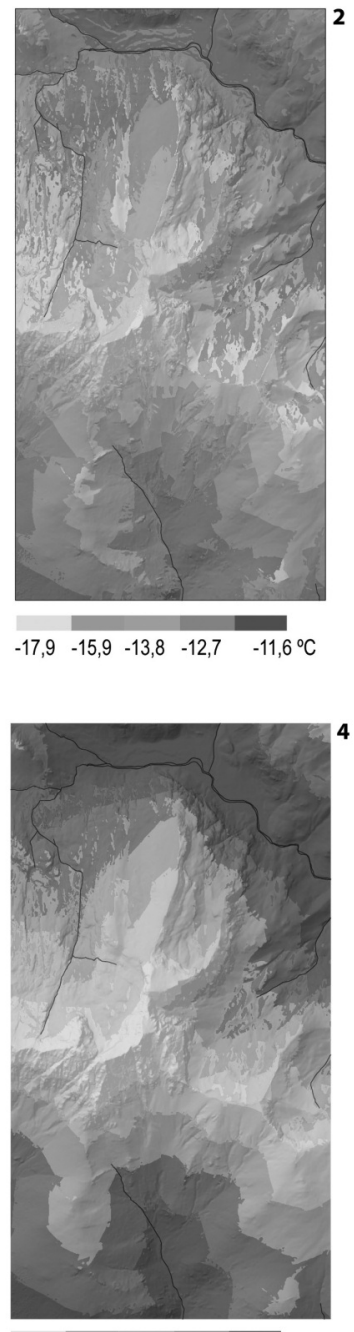

$\begin{array}{lllll}3,4 & 5 & 6 & 6,8 & 7,6^{\circ} \mathrm{C}\end{array}$ $325 \mathrm{~m}$.

Fuente: elaboración propia a partir de los datos proporcionados del SIGA (2013) y MDE a partir de datos LIDAR (IGN, 2013). 


\section{Los parámetros climáticos}

Pluviometría (cuadro 2, 2.1 y figura 3.1) y espesor de nieve (cuadro 2, 2.3)

Los cordales más elevados y su prolongación en los contrafuertes de la vertiente norte (crestería de la Cola del Curavacas) concentran los máximos de las medias anuales $(2.883,4 \mathrm{~mm})$ registrándose mínimos en la vega del Carrión $(1.176,2 \mathrm{~mm})$. En su régimen interanual los máximos principales son en noviembre $(370,3 \mathrm{~mm})$ y febrero $(286,8 \mathrm{~mm})$ con dos repuntes secundarios en mayo $(233,3 \mathrm{~mm})$ y septiembre $(170,6 \mathrm{~mm})$. Los mínimos son especialmente acusados en agosto $(51,7 \mathrm{~mm})$. En ningún caso existe una evapotranspiración negativa debido al alto excedente acumulado (cuadro 1.4). Los espesores de nieve, pese a lo sesgado de los datos y a su ubicuidad temporal, muestran importantes acumulaciones en marzo $(113,1-178,8 \mathrm{~cm})$ asociadas a las intensas nevadas de enero y febrero en las temporadas 92-93 y 93-94. Máximos secundarios se registran en enero $(113,1 \mathrm{~cm})$ y tardíos extraordinarios pero de escasa persistencia en mayo $(175,2 \mathrm{~cm})$.

\section{Temperatura (cuadro 2, 2.2 y figura 3.2 y 3.4) e insolación (cuadro 2, 2.4)}

Considerando las medias mensuales del mes más cálido y del más frío se registran valores mínimos en julio de $11,7^{\circ} \mathrm{C}$ y máximos de $16,2^{\circ} \mathrm{C}$; en el mes más frío (enero) los datos obtenidos oscilan entre $-6,1^{\circ} \mathrm{C}$ y 0,4 . En lo referente a las medias de las mínimas absolutas mensuales enero registra mínimos de $-18,5^{\circ} \mathrm{C}$ y máximos de $-11,7^{\circ} \mathrm{C}$ con registros para julio entre $-4,8^{\circ} \mathrm{C}$ y $1,5^{\circ} \mathrm{C}$. Los registros térmicos, debido al peso adquirido por la variable orientación en el cálculo, dibujan el conjunto de umbrías y sombras topográficas de la arista culminante y del cresterío de La Cola del Curavacas. Los máximos se registran en los amplios llanos del valle del Carrión y en el umbral de los Escalones. La insolación, computada en horas, presenta mínimos en el solsticio invernal. En esta fecha una amplia superficie de la vertiente septentrional se considera en la categoría de 0 horas y gran parte del piedemonte sur se incluye en el intervalo entre 5 y 7 horas. Por el contrario en el solsticio vernal la mayor parte del macizo se considera entre 10 y 14 horas. Por último en los equinoccios gran parte del el intervalo dominante se sitúa entre 7 y 11 horas frente a una menor representatividad del grupo entre 0 y 6.

Índices bioclimáticos (cuadro 2, 2.6 y figura 3.3)

Se han obtenido cuatro pisos bioclimáticos según la clasificación de Rivas Martínez ${ }^{5}$ siendo el mejor representado el Subalpino con un horizonte inferior 
CuAdro 2

SÍNTESIS DE DATOS CLIMÁTICOS

\subsection{PLUVIOMETRÍA (mm)}

\begin{tabular}{ccccccccccccc}
\hline \multicolumn{2}{c}{ Noviembre } & \multicolumn{2}{c}{ Febrero } & \multicolumn{2}{c}{ Mayo } & \multicolumn{2}{c}{ Agosto } & \multicolumn{2}{c}{ Septiembre } & \multicolumn{2}{c}{ Anual } \\
\hline $\min$ & $\max$ & $\min$ & $\max$ & Min & $\max$ & $\min$ & $\max$ & $\min$ & $\max$ & $\min$ & $\max$ \\
\hline 122,8 & 370,3 & 95,9 & 286,8 & 103 & 233,3 & 37,2 & 51,7 & 64,3 & 170,6 & $1.176,2$ & 2883,4 \\
\hline
\end{tabular}

\subsection{TERMOMETRÍA $\left({ }^{\circ} \mathrm{C}\right)$}

\begin{tabular}{cccccccc}
\hline \multicolumn{2}{c}{ Julio (1) } & \multicolumn{2}{c}{ Enero (1) } & \multicolumn{2}{c}{ Julio (2) } & \multicolumn{2}{c}{ Enero (2) } \\
\hline $\min$ & $\max$ & $\min$ & $\max$ & $\min$ & $\max$ & $\min$ & $\max$ \\
\hline 11,7 & 16,2 & $-6,1$ & 0,4 & $-4,8$ & 1,5 & $-18,5$ & $-11,7$ \\
\hline
\end{tabular}

1.3 ESPESOR DE NIEVE $(\mathrm{cm})$

\begin{tabular}{ccccc}
\hline $\begin{array}{c}\text { 22-23 marzo } \\
91-92\end{array}$ & $22-23$ marzo & $26-27$ marzo & 28-29 marzo & $4-5$ mayo \\
\hline 113,1 & $93-94$ & $93-94$ & $92-93$ & $92-93$ \\
\hline
\end{tabular}

1.4 EVAPOTRANSPIRACIÓN POTENCIAL (THORNTHWAITE, mm)

\begin{tabular}{ccccccccccccc}
\hline \multicolumn{2}{c}{ Noviembre } & \multicolumn{2}{c}{ Febrero } & \multicolumn{2}{c}{ Mayo } & \multicolumn{2}{c}{ Agost } & \multicolumn{2}{c}{ Septiembre } & \multicolumn{2}{c}{ Anual } \\
\hline $\min$ & $\max$ & $\min$ & $\max$ & $\min$ & $\max$ & $\min$ & $\max$ & $\min$ & $\max$ & $\min$ & $\max$ \\
\hline 19.1 & 24.9 & 0 & 7.8 & 33.7 & 60 & 63.2 & 96.9 & 53.2 & 71.1 & 362,4 & 604,5 \\
\hline
\end{tabular}

1.5 INSOLACIÓN (máximo en horas)

\begin{tabular}{ccc}
\hline Solsticio de invierno & Solsticio de verano & Equinoccios de primavera y otoño \\
\hline 7 & 14 & 11 \\
\hline
\end{tabular}

1.6 PISOS BIOCLIMÁTICOS (referidos a RIVAS MARTÍNEZ, 1984)

\begin{tabular}{cccccccc}
\hline \multicolumn{2}{c}{$\begin{array}{c}\text { Altimontano } \\
(20,13 \text { has })\end{array}$} & \multicolumn{2}{c}{$\begin{array}{c}\text { Subalpino inferior } \\
(322,4 \text { has })\end{array}$} & $\begin{array}{c}\text { Subalpino superior } \\
(1140,7\end{array}$ & \multicolumn{2}{c}{$\begin{array}{c}\text { Alpino inferior } \\
(138,6 \text { has })\end{array}$} \\
\hline $\min$ & $\max$ & Min & $\max$ & $\min$ & $\max$ & $\min$ & $\max$ \\
\hline 95,4 & 60 & 60 & 0 & 0 & -40 & -40 & $-77,8$ \\
\hline
\end{tabular}


reducido (322, 4 has) mientras que el superior es el mejor representado (1140, 7 has.). Por otra parte el altimontano, dado el rigor térmico y la altimetría del área de trabajo, es el de menor extensión $(20,13$ has) y en gran parte está condicionado por la proximidad de su valor mínimo al límite inferior del Subalpino $(95,4)$. El Alpino inferior aparece representado en las crestas y sectores culminantes con un valor máximo $(-77,8)$ cercano al límite inferior del Alpino superior (-80). Es, por tanto, un régimen caracterizado por máximos precipitacionales principalmente invernales y con repuntes secundarios en primavera.

\section{La caracterización de las comunidades vegetales}

Los contrastes altitudinales, la diversidad de orientaciones y la presencia de un mosaico de roquedos, pedreras y lomas son algunos de los condicionantes fisiomorfológicos más determinantes para la distribución de la vegetación

\section{Cuadro 1}

SINTESIS DE LAS COMUNIDADES VEGETALES MUESTREADAS

\begin{tabular}{|c|c|c|c|}
\hline Matas y patches arbolados y & Matorrales & \multicolumn{2}{|c|}{ Pastizales y herbazales } \\
\hline \multirow{2}{*}{$\begin{array}{l}\text { Matas de Quercus pyrenaica } \\
\text { (Altimontano superior-Subalpino inferior) }\end{array}$} & \multirow{2}{*}{$\begin{array}{l}\text { Urcedas higrófilas de Erica arborea } \\
\text { (Altimontano superior-Subalpino superior) }\end{array}$} & \multirow{2}{*}{$\begin{array}{l}\text { Cervunales } \\
\text { (Subalpino inferior- Subalpino } \\
\text { superior) }\end{array}$} & ruderalizados con Erica tetralix \\
\hline & & & higro-umbróflos con Gentiana lutea \\
\hline \multirow{2}{*}{$\begin{array}{l}\text { Abedulares de Betula alba } \\
\text { (Altimontano superior-Subalpino inferior) }\end{array}$} & \multirow{2}{*}{$\begin{array}{l}\text { Genistedos de Genista florida } \\
\text { (Altimontano superior-Subalpino inferior) }\end{array}$} & \multirow{2}{*}{$\begin{array}{c}\text { Pastizales de Festuca eskia } \\
\text { (Subalpino inferior-Alpino inferior) }\end{array}$} & $\begin{array}{l}\text { sustratos solifluidales o gelifluidales } \\
\text { móviles con Agrostis rupestris }\end{array}$ \\
\hline & & & $\begin{array}{l}\text { rellanos y repisas crioturbados con } \\
\text { Galium saxatile }\end{array}$ \\
\hline & \multirow{2}{*}{$\begin{array}{l}\text { Piornales de Cytisus oromediterraneus } \\
\text { (Subalpino inferior- Subalpino superior) }\end{array}$} & \multirow{2}{*}{$\begin{array}{c}\text { Herbazales rupicolas } \\
\text { pluriespecíficos } \\
\text { (Subalpino superior-Alpino inferior) }\end{array}$} & heliófilos con Juncus trifidus \\
\hline & & & umbróflos con Murbeckiella boryi \\
\hline & \multirow[t]{2}{*}{$\begin{array}{l}\text { Arandaneras de Vaccinium uliginosum } \\
\text { (Subalpino superior) }\end{array}$} & \multirow{2}{*}{$\begin{array}{c}\text { Pastizales sobre suelos } \\
\text { crioturbados } \\
\text { (Subalpino superior-Alpino inferior) }\end{array}$} & $\begin{array}{l}\text { rellanos con Deschampsia flexuosa } \\
\text { subsp. flexuosa }\end{array}$ \\
\hline & & & $\begin{array}{l}\text { rellanos pedregosos con Aira } \\
\text { caryophyllea subsp. multiculmis }\end{array}$ \\
\hline & & Pastizales psicroxerófilos & $\begin{array}{l}\text { pavimentos subverticales con } \\
\text { Festuca iberica }\end{array}$ \\
\hline & & & glerícolas con Luzula nutans \\
\hline
\end{tabular}


del Curavacas. A lo anterior se une la desaparición de las prácticas agroganaderas tradicionales, decisivas en las activas dinámicas de avance del matorral y matas arboladas del pie de peña. Los 18 muestreos (anexo 1) realizados se repartieron entre el Subalpino inferior y el Alpino inferior, diferenciándose tres tipos de comunidades vegetales: pastizales, matorrales y matas rodales de arbolado (cuadro 3 y figura 7). Entre ellas se han analizado con mayor detalle aquellas comunidades que hemos considerado más representativas.

\section{Pastizales $y$ herbazales}

Los pastizales son la comunidad vegetal más abundante y variada. Se han muestreados cuatro tipos: herbazales rupícolas, patches herbáceos sobre suelos crioturbados, pastos psicroxerófilos de cumbres y crestas y cervunales y herbazales rupícolas.

Los herbazales de Festuca eskia sobre litologías conglomeráticas alcanzan su óptimo entre el Subalpino superior y el Alpino inferior y están bien representados en macizos cercanos como Peña Prieta (Allende, 2007). Dentro de la cartografía se incluyen en el grupo de herbazales rupícolas asociados a pavimentos, pedregales y repisas sobre conglomerados. En el primer caso (inven. 1, figura 4.1) muestran, respecto a las muestreadas en Peña Prieta, una mayor termofilia (Allende, 2007). Festuca eskia domina la comunidad (60) ${ }^{6}$ acompañada por Agrostis rupestris (10), Campanula scheuchzeri, Conopodium majus subsp. majus, Cytisus oromediterraneus, Gentiana lutea, Jasione sessiliflora (1), Rumex suffruticosus (1), Sedum brevifolium y Silene ciliata. La segunda facies se localiza en las canales más elevadas colonizando rellanos de roquedos (inven. 2, 3 figura 4.2). Como especies comunes, al igual que en el caso anterior, Festuca eskia (60 y 80) y Luzula nutans (20, 5 y 10), típicas de pastos acidófilos petranos (inven. 5). Su cohorte posee elementos propios de medios rupícolas con Galium saxatile (10), Alchemilla saxatilis, Cryptogramma crispa, Deschampsia flexuosa (5), Jasione laevis, Leontodon hispidus subsp. hispidus y Festuca iberica (5), Galium saxatile (5), Deschampsia flexuosa (5), Anthoxanthum odoratum (5) y Campanula scheuchzeri. En la vertiente norte, ante la pérdida de verticalidad del roquedo (inven. 5; Subalpino superior), la comunidad se nutre de elementos típicos de pastos psicroxerófilos frescos en las fisuras de pavimentos conglomeráticos con Festuca iberica (10), Paronychia kapela subsp.

\footnotetext{
${ }^{6}$ Se muestra entre paréntesis el porcentaje ocupado por la especie respecto al resto de especies inventariadas del muestreo.
} 
serpyllifolia (10), Poa bulbosa (5), Rumex acetosella subsp. angiocarpus (1), Sedum dasyphyllum subsp. dasyphyllum (5), Silene nutans subsp. nutans (1), Nardus stricta (1), Festuca rubra (5), Jasione laevis (1) y Agrostis delicatula (5).

Dentro de los herbazales rupícolas existen microhábitats asociados a la duración de la innivación, la exposición a los vientos y a la dimensión y pendiente de sus fisuras diferenciándose dos facies. La primera, heliófila, ocupa las fisuras y pavimentos subhorizontales más expuestos; la segunda, umbrófila, coloniza las fisuras verticales más profundas. La faciación heliófila tiene sus mejores representaciones en el Alpino inferior del Curavacas. La facies está dominada por Juncus trifidus (inven 11; figura 4.5) que coloniza un variado mosaico de repisas, pedregales y pavimentos subhorizontales. En estas comunidades las especies responden a las condiciones microtopográficas que impone la inestabilidad del sustrato. Las repisas más amplias son ocupadas por Festuca eskia (5) en mosaico con especies típicas de pastos alpinos y subalpinos psicroxerófilos petranos como Oreochloa disticha subsp.blanka (20), Deschampsia flexuosa (10), Agrostis capillaris (10) y Luzula nutans, en alternancia con elementos característicos de roquedales y pedregales expuestos como Sedum candollei, Sempervivum vicentei subsp. cantabricum, Silene ciliata, Armeria cantabrica (1) y Alchemilla saxatilis. Los roquedos verticales sobre conglomerados del Subalpino superior (inven 12; figura 5.5) albergan comunidades herbáceas de escasa cobertura dominadas por taxones propios de fisuras y repisas umbrosas como Murbeckiella boryi (1), el endemismo ibérico Saxifraga pentadactylis subsp. wilkkommiana (1), Alchemilla saxatilis (1), Armeria cantabrica (1), Campanula scheuchzeri, Cryptogramma crispa (1), Festuca iberica (1) y Polypodium vulgare.

Sobre pequeños umbrales estructurales donde son frecuentes los empapamientos temporales que facilitan procesos de crioturbación se localiza un conjunto de herbazales de composición heterogénea. Lejos de formar comunidades de cierta densidad como los de Festuca eskia, conforman mosaicos que se densifican únicamente en las zonas con sustratos más favorables. Se instalan en el Subalpino superior intercalados entre los dominados por Festuca eskia siendo la especie dominante Deschampsia flexuosa subsp. flexuosa (inven. 6) acompañada de elementos propios de fisuras húmedas y medios crioturbados. Destacan Alchemilla saxatilis (1), Agrostis delicatula (10), Juncus trifidus (5) y Luzula nutans (20). A éstos se le suman taxones propios de medios con cierta inestabilidad superficial como Festuca iberica (10), Jasione laevis, Sedum brevifolium (1) y Silene nutans subsp. nutans (5) e inclusive otros ligados a la existencia de roquedos como Juniperus communis subsp. alpina. Dentro de la misma tipología se incluyen los pastizales terofíticos sobre rella- 
nos pedregosos sombreados utilizados como sesteadero estival por el ganado (inven. 7). Domina Aira caryophyllea subsp. multiculmis (20) acompañada de Cytisus oromediterraneus (10), Erica arborea (5), Genista florida, Luzula nutans (1), Rumex suffruticosus (10) y Sedum brevifolium.

\section{FIGURA 4}

\section{PASTIZALES Y HERBAZALES}
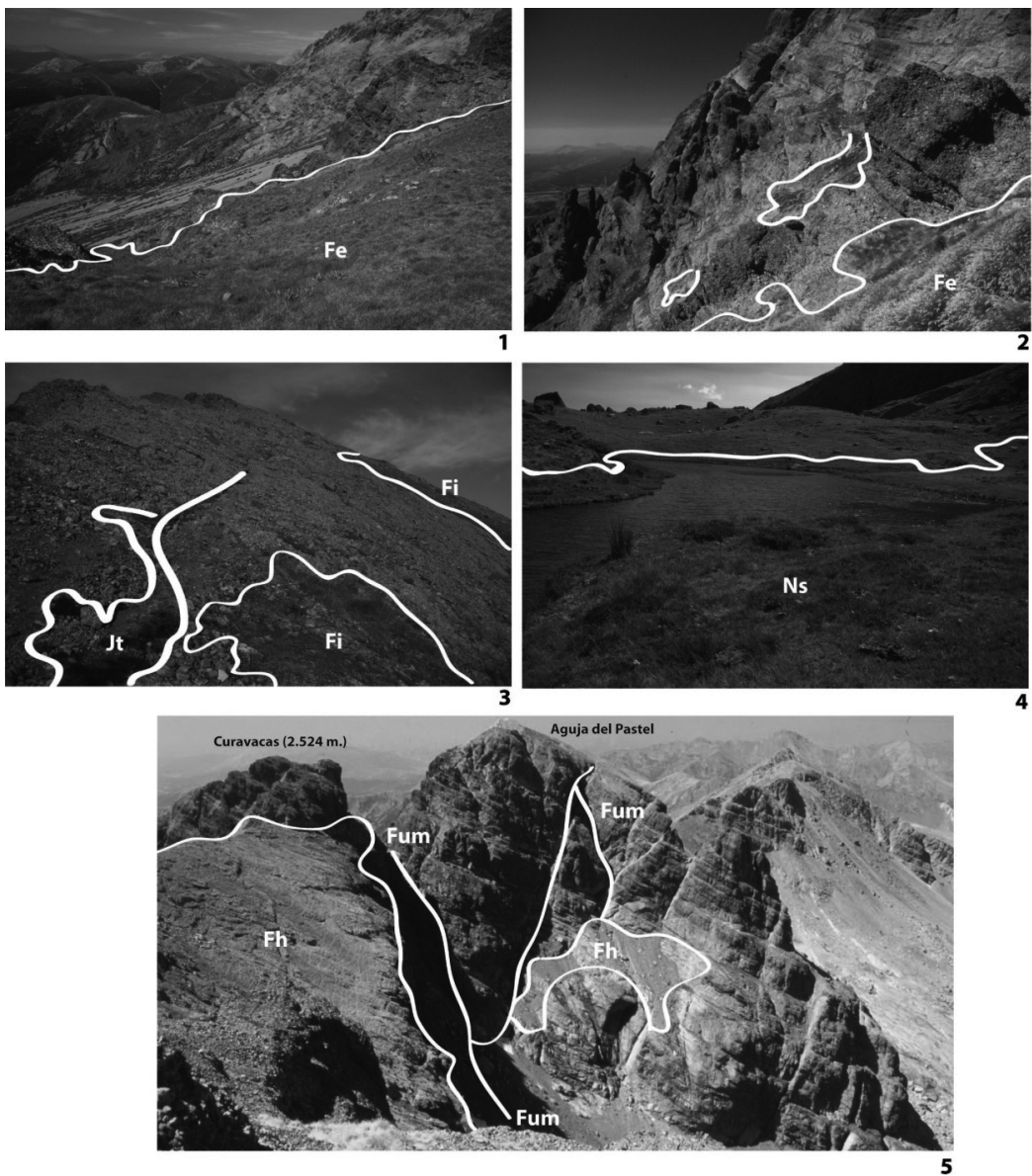

De Festuca eskia en pedreras conglomeráticas (1) y repisas (2) en la vertiente a Vidrieros; pastos psicroxerófilos sobre pavimentos conglomeráticos de Festuca iberica (Fi) y Juncus trifidus (Jt) cerca de la cumbre (3); cervunales (Ns) en el hoyo muerto, vertiente norte (4); distribución de las faciaciones heliófila (Fh) y umbrófila $(\mathrm{Fu})$ de los herbazales rupícolas en la cumbre y la aguja del pastel (5) 
Los ventisqueros, collados y crestas culminantes desde el Subalpino superior al Alpino inferior están colonizados por pastos psicroxerófilos. Son comunidades en su mayor parte de naturaleza petrana sobre sustratos fragmentados por la acción de los ciclos de hielo-deshielo y con elevada xericidad ambiental y edáfica. Se localizan en el Subalpino Superior (inven. 8, figura 5.3) y forman un mosaico dentro de los limitados rellanos de los pavimentos subverticales culminantes. En este caso el estrato graminoide está compuesto por Festuca iberica (30) asociada con Agrostis capillaris (1), Deschampsia flexuosa (10), Oreochloa disticha subsp. blanka (5) y con especies netamente quinófilas y adaptadas a la crioturbación como Armeria cantabrica (1) y Juncus trifidus (1). A éstas les acompañan taxones de pastos pedregosos como Luzula nutans (5) y adquieren gran interés los comunidades rupícolas con Cryptogramma crispa (1), Minuartia recurva, Sedum brevifolium, Sedum candollei, Alchemilla saxatilis, Sempervivum vicentei subsp. cantabricum y Silene nutans subsp. nutans. Estos pastos son comparables por su morfología y ecología a los muestreados sobre las crestas culminantes de Peña Prieta-Tres Provincias de Festuca iberica (Subalpino superior, $2.411 \mathrm{~m}$ ), Deschampsia flexuosa (Subalpino superior, $2.257 \mathrm{~m}$ ) o de Orechloa disticha subsp. blanka (Alpino inferior, $2.384 \mathrm{~m}$ ). En las crestas del Alpino inferior (inven. 4) a una menor innivación se une un aumento en la dimensión de las repisas y de la pedregosidad superficial. Estos condicionantes favorecen la presencia de una comunidad propia de medios glerícolas con Luzula nutans (20) y Festuca eskia (20) acompañadas por taxones propios de pastos psicroxerófilos con cierta nitrificación superficial y elementos típicamente rupícolas como Festuca iberica (10), Meum athamanticum (2), Galium saxatile (1), Aira caryophyllea subsp. multiculmis (5), Pilosella officinarum (1), Deschampsia flexuosa (1), Trisetum flavescens flavescens (1), Thymus praecox subsp. polytrichus (10), Minuartia recurva (1), Agrostis capillaris, Alchemilla saxatilis, Campanula scheuchzeri, Gentiana lutea, Jasione laevis, Juncus trifidus, Juniperus communis subsp. alpina, Murbeckiella boryi, Rumex suffruticosus, Sedum brevifolium, Silene nutans subsp. nutans y Solidago virgaurea var. minuta.

Los cervunales de Nardus stricta son comunidades ligadas a la presencia de agua, bien en surgencias fontinales, áreas lagunares o a las aguas de deshielo de los neveros. Se diferencian dos facies incluidas en la tipología establecida por Nava (1988): ruderalizada e higro-umbrófila. En las áreas lagunares del Subalpino superior represadas por cordones morrénicos, alcanzan su óptimo las comunidades higrófilas ruderalizadas (inven 9, figura 4.4). Están condicionadas por una intensa nitrificación debida a la presencia de ganado en la época estival (descanso y pastadero estival). Todos estos elementos influyen 
en la gran heterogeneidad de la comunidad, con elementos característicos de áreas húmedas entre los que encontramos Nardus stricta (30), Carex echinata (5), Carex nigra (10), Gentiana pneumonanthe (1), Juncus squarrosus (5), Erica tetralix (10) y Juncus effusus, frente a taxones de mayor plasticidad ecológica como Agrostis castellana var. castellana (5), Deschampsia flexuosa (5), Festuca rubra (20), Luzula nutans (5), Pimpinella major (1), Calluna vulgaris, Galium saxatile, Cryptogramma crispa, Jasione laevis, Juniperus communis subsp. alpina, Rumex acetosella subsp. angiocarpus, Sedum brevifolium y Vaccinium myrtillus. En las áreas con fuertes pendientes y derrames de ladera se localizan cervunales higro-umbrófilos ruderalizados (inven 10). Abundan los elementos típicos de pastos petranos y herbazales megafórbicos con Festuca iberica (10), Festuca rubra (10), Gentiana lutea (30), Campanula rotundifolia subsp. hispanica, Conopodium majus subsp. majus y Cryptogramma crispa, y otros característicos de medios húmedos con Erica tetralix (5), Moehringia trinervia (1), Nardus stricta (40), Saxifraga stellaris (1) y Viola palustris.

\section{Los matorrales}

La segunda comunidad mejor representada son las etapas seriales propias del abandono del pastadero a causa de continuos fuegos de suelo que, una vez acabada la práctica, favorecen la progresión de formaciones leñosas. Claramente seriados en altura diferenciamos, por su porte, los de tipo arborescentearbustivo (genistedos y escobonales), arbustivo (piornales), subarbustivo (urcedas) y rastrero (arandaneras).

Los genistedos y escobonales (figuras 5.1 y 5.2) arborescente-arbustivos poseen sus mejores densidades sobre las areniscas y pizarras del Altimontano en vertientes modeladas por la solifluxión. En este caso están ligados a la amplia solana meridional donde Genista florida alcanza, ya sobre materiales conglomeráticos, los niveles inferiores del Subalpino inferior. Ocupan áreas donde las rozas para la obtención de terrenos de siembra o las quemas para la apertura de pastizales fueron un hecho frecuente hasta mediados del siglo xx. Se definen como densas comunidades retamoides pluriespecíficas. El genistedo muestreado presenta elementos de transición hacia los piornales de cumbres (inven. 13), típicos del Subalpino, como así se manifiesta con la presencia como acompañante de Cytisus oromediterraneus (1). La pedregosidad superficial resulta determinante en la aparición de taxones asociados a medios petranos como Agrostis capillaris, Festuca iberica (1), Rumex suffruticosus (1) y Sedum brevifolium; así como de pastos y sotobosques húmedos con Aira car- 
yophyllea subsp. multiculmis (5), Trisetum flavescens subsp. flavescens (5) o Conopodium majus subsp. majus y, debido a la canalización de flujos de agua dentro del pedregal, de Erica arborea (10).

En el Subalpino son frecuentes comunidades de escasa cobertura dominadas por las especies de naturaleza subarbustiva y rastrera. Son formaciones pobres en taxones que colonizan collados y laderas pedregosas con prolongada innivación. Diferenciamos las fitomorfologías subarbustivas de Cytisus oromediterraneus y las rastreras de Vaccinium uliginosum. Los piornales de Cytisus alcanzan, al amparo de la amplia solana meridional y sobre pedreras conglomeráticas parcialmente fosilizadas, el horizonte superior del Subalpino (inven. 14). Constituyen formaciones con un estrato subarbustivo dominado por Cytisus oromediterraneus (40), al que acompañan, puntualmente, Erica arborea y Juniperus communis subsp. alpina. A las anteriores se suman, en el herbáceo, especies típicas de pedregales y pastos petranos con Festuca eskia (20), Aira caryophyllea subsp. multiculmis (10), Festuca iberica (5), Trisetum flavescens subsp. flavescens (5), Agrostis delicatula (1), Luzula nutans (1) y Rumex suffruticosus (1) y, al amparo de grandes bloques, Cryptogramma crispa. Estas formaciones están en proceso de consolidación, como así lo pone de manifiesto su porte reducido y su disposición en rodales, sobre antiguos pastaderos de ovino.

Sobre la vertiente orientada al Norte y en la incluida en el Subalpino superior, se desarrollan densos arbustedos rastreros de Vaccinium uliginosum (inven. 16). Colonizan sustratos crioturbados y laderas modeladas por la solifluxión. El arándano cubre el 40\% acompañado de Juncus trifidus (20), Dechampsia flexuosa (30), Luzula nutans (5), Silene nutans subsp. nutans (1), Jasione laevis, Leontodon hispidus subsp. hispidus, Solidago virgaurea var. minuta, Campanula scheuchzeri y Gentiana lutea, todas ellas especies indicadoras de pastos petranos frescos. A éstas les acompaña Erica tetralix, taxon que pone de manifiesto la presencia de agua superficial procedente de las canales superiores.

Señalar la presencia puntual de urcedas arbustivas higrófilas de Erica arborea (inven. 15; figura 5.1 y 5.2) cuyo óptimo se alcanza sobre los suelos turbosos desde el Altimontano al Subalpino. Sus mejores representaciones se localizan al pie del afloramiento conglomerático, en áreas donde se concentra la escorrentía procedente de las vertientes altas (inven. 2). Son una formación que aparece con cierta frecuencia en antiguas cubetas de excavación glaciar, afectadas por procesos solifluidales y colmatadas de turba. La solifluxión favorece el típico aspecto lobulado de la comunidad con gran diversidad de microambientes. 
FigURA 5

MATORRALES
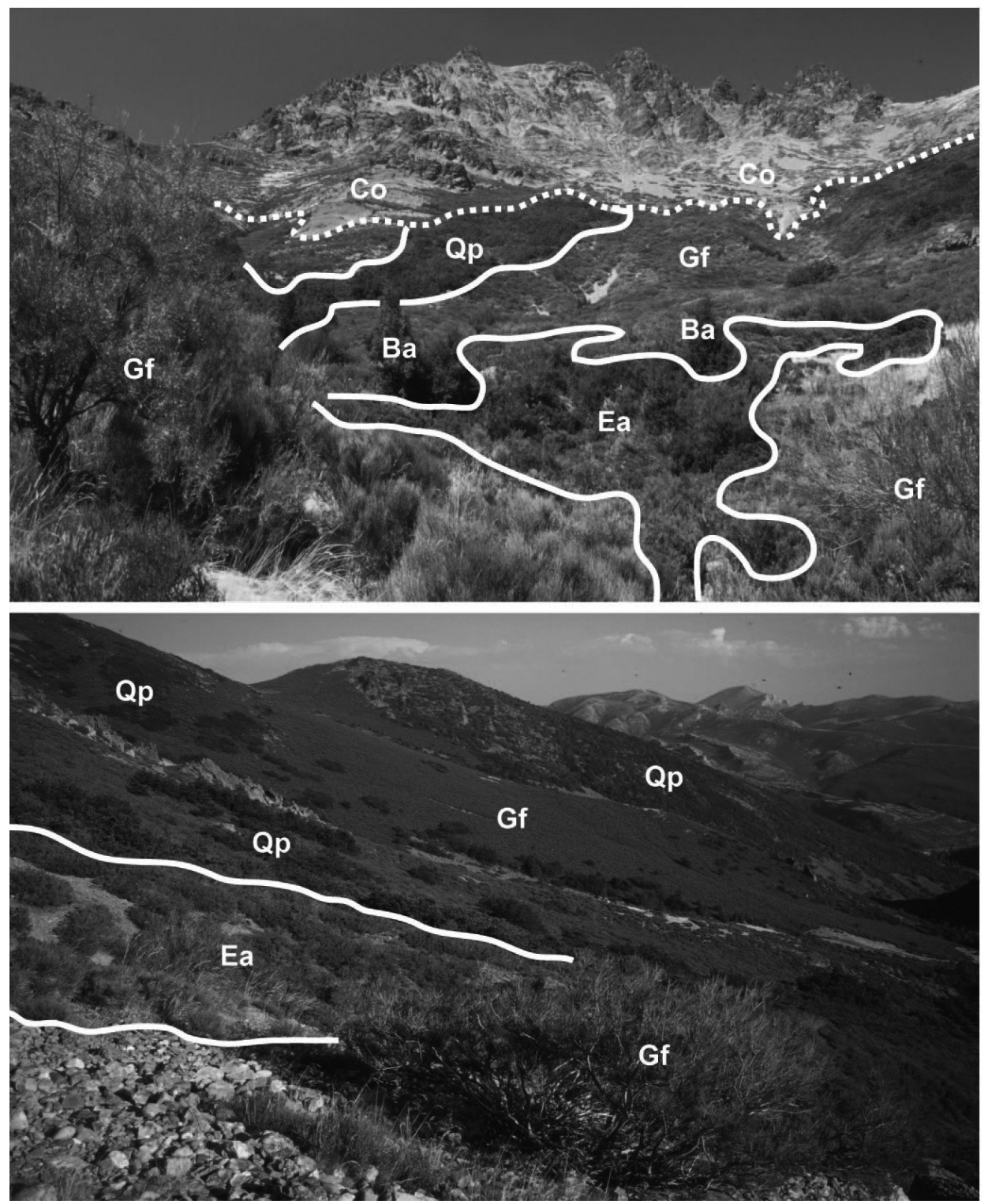

De Genista florida (Gf), Erica arborea (Ea) y Cytisus oromediterraneus (Co) en la vertiente meridional (especies arbóreas y arborescentes: Betula alba-Ba- y Quercus pyrenaica-Qp-).

Estudios Geográficos, Vol. LXXVI, 278, pp. 7-38, enero-junio 2015 ISSN: 0014-1496, eISSN: 1988-8546, doi: 10.3989/estgeogr.201501 


\section{Matas de Quercus pyrenaica y abedulares}

Los robledales de melojo se localizan sobre coluviones y roquedos y conforman una mata arborescente de escaso desarrollo y muy fragmentada con portes de mata arborescente o arbustiva con activas dinámicas de progresión (figura 6). La estructura de estas masas forestales es el resultado de las quemas para la obtención de pastos y de las podas para leñas y ramón (inven. 17). Quercus pyrenaica cubre el 45\% (el total del arborescente) de la cabida acompañado, en el arbustivo y subarbustivo, de matorrales típicos de formaciones de sustitución con Cytisus oromediterraneus (5) y Genista obtusiramea; así como de especies que requieren cierta humedad edáfica, canalizada a través de las pedreras conglomeráticas, como Erica arborea (20). En el herbáceo se mezclan especies propias de pastos frescos, como Arrhenatherum elatius elatius var bulbosum (5), con otras de pastos y robledales petranos (Aira caryophyllea subsp. multiculmis -20-, Festuca iberica -5- y Conopodium majus subsp. majus).

FIGURA 6

\section{MATAS ARBORESCENTES}
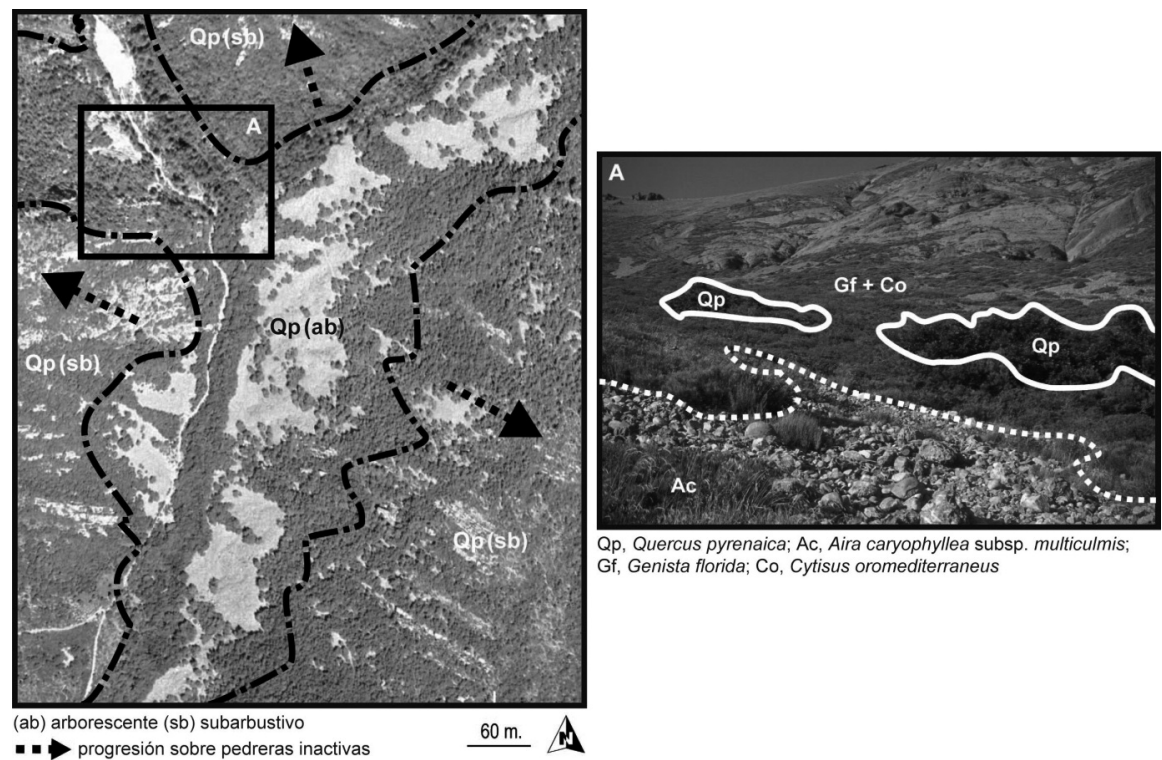

Qp, Quercus pyrenaica; Ac, Aira caryophyllea subsp. multiculmis $\mathrm{Gf}$, Genista florida; Co, Cytisus oromediterraneus

De Quercus pyrenaica en la vertiente meridional y configuración de la mata en el contacto con la "peña". Subarbóreo Qp (sb) y arborescente Qp (ab) y configuración detallada de la mata en su límite superior. (Ortofotografía 1:5.000 PNOA, IGN, 2009) 


\section{FIGURA 7}

\section{MAPA DE VEGETACIÓN}

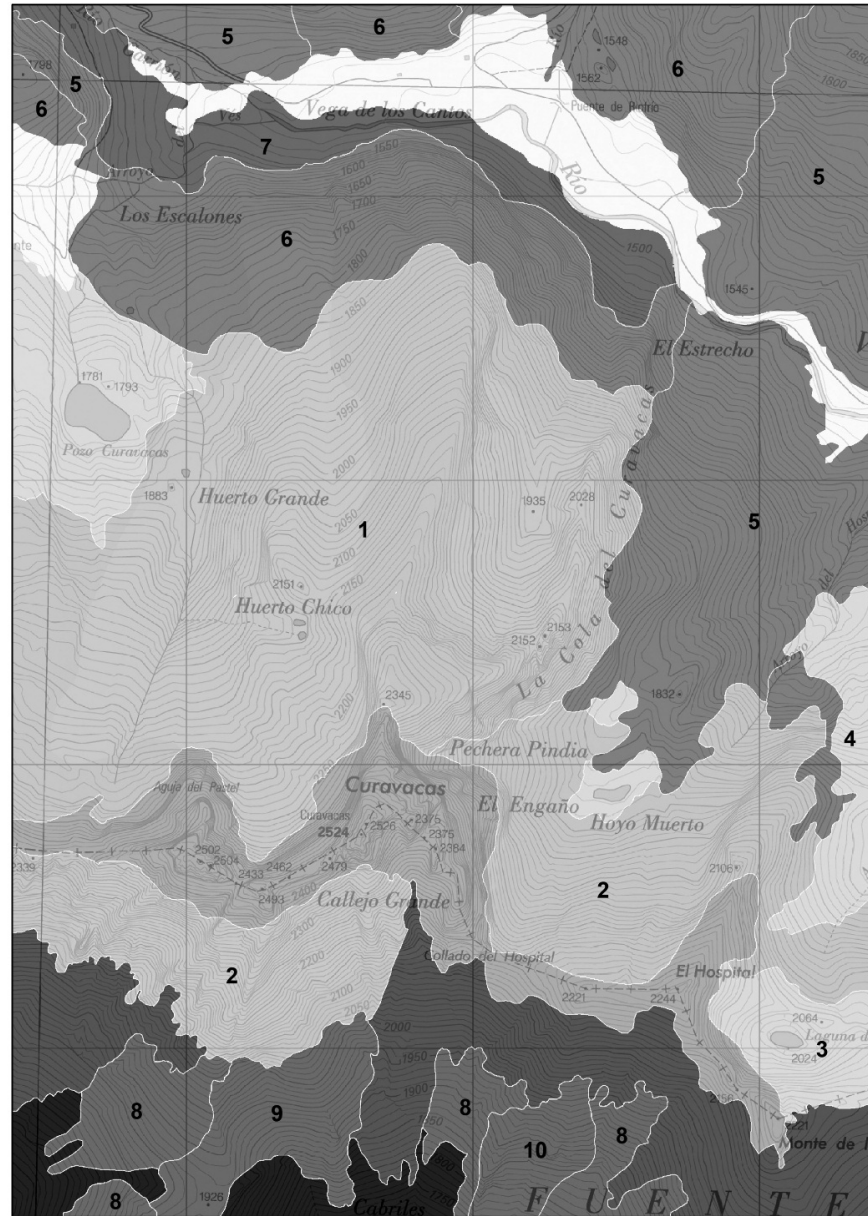

\section{$250 \mathrm{~m}$.}

Pastizales psicroxerófilos de cumbres y crestas

Herbazales rupicolas con Festuca eskia (1. sobre pavimentos conglomeráticos ; 2 . sobre pedreras activas y repisas) Pastizales de Festuca rubra y Nardus stricta

Mosaicos (3. de herbazales rupicolas y cervunales; 4. de Calluna vulgaris, Erica australis y Nardus stricta)

Piomales de Cytisus oromediterraneus sobre pedreras activas

Brezales de Calluna vulgaris y Erica australis (5. sobre vertientes; 6 . sobre roquedos y pavimentos conglomeráticos)

Genistedos (7. con Erica australis y Calluna vulgaris; 8 . con Cytisus oromediterraneus sobre pedreras

parcialmente fosilizadas; 9 . con Cytisus oromediterraneus sobre vertientes rocosas; 10 . sobre vertientes rocosas)

Robledales de Quercus pyrenaica con Genista florida

Planimetría MTN25 IGN, 2013

Estudios Geográficos, Vol. LXXVI, 278, pp. 7-38, enero-junio 2015 ISSN: 0014-1496, eISSN: 1988-8546, doi: 10.3989/estgeogr.201501 
Relacionados con zonas higroturbosas o con formaciones seriales del timberline boscoso sobre medios ácidos se desarrollan abedulares de Betula alba. Alcanzan su óptimo en los niveles superiores del Altimontano e inferiores del Subalpino. Como ejemplo de este tipo de formación tomamos un abedular localizado en la vertiente meridional del Curavacas (inven. 18), en el Subalpino inferior. El abedular presenta una estructura dominada por Betula que alcanza el $40 \%$ de la cabida cubierta, acompañado de especies características de tollares acidófilos y comunidades fontinales como Erica tetralix (10), Carex echinata (1), Carum verticillatum (2), Ranunculus longipes (1), Juncus articulatus (1), Juncus effusus (1), Parnassia palustris subsp. palustris (1), Juncus squarrosus, Lotus pedunculatus, Wahlenbergia hederacea así como elementos propios de pastos frescos y orlas forestales con Festuca rubra (10), Holcus lanatus (10), Agrostis castellana var. castellana (10), Arrhenatherum elatius elatius var. bulbosum (2), Briza media (1), Potentilla erecta (5), Prunella hastifolia (1), Salix atrocinerea (5), Ranunculus bulbosus bulbosus var. hispanicus (2), Trifolium pratense pratense var. pratense (1), Calluna vulgaris, Chamaespartium sagittale, Corylus avellana, Cytisus scoparius scoparius, Erica arborea, Genista florida, Plantago media, Pteridium aquilinum subsp. aquilinum y Stellaria alsine.

\section{La fusión de los muestreos de vegetación y los datos climáticos}

El análisis resultante de la intersección entre las especies identificadas en los muestreos y los parámetros climáticos modelizados ha permitido la discriminación de agrupaciones de especies mediante el método de las distancias euclídeas. Este proceso se realizó sobre un total de 69 variables abióticas que, para una mejor interpretación, se agruparon por grado de afinidad en 5 conjuntos: térmicas y bioclimáticas, morfofisiográficas, insolación directa en los solsticios y equinoccios (horas medias totales) y nivopluviométricas. Del análisis de las relaciones de afinidad se obtuvieron tres grupos para cada uno de los conjuntos, salvo en el caso de las variables bioclimáticas en las que se identificaron dos (cuadro 4).

Dentro de las variables térmicas el primer grupo $(48)^{7}$ incluye robledos, matorrales supraforestales y comunidades subarbustivas heliófilas de arroyos (Trifolium pratense pratense var. pratense/Vaccinium myrtillus). Las temperaturas medias oscilan entre 8,3 y $6,9^{\circ} \mathrm{C}$ y las medias de las mínimas del mes más

\footnotetext{
${ }^{7}$ Número de especies por agrupación.
} 
frío alcanzan los $-13,4^{\circ} \mathrm{C}$. Se extiende por toda la solana superior orientada a Vidrieros, desde el Altimontano al Subalpino inferior. Los valores más bajos se alcanzan en el grupo 2 (11) que incluye las comunidades vegetales asociadas a los roquedos del Alpino (Sedum candollei/Armeria cantabrica) destacando las mínimas absolutas del mes más frío con $-21,1^{\circ} \mathrm{C}$ o las medias anuales de $3,9^{\circ} \mathrm{C}$. EL tercer grupo (46) se asocia a las comunidades vegetales subalpinas y de los horizontes inferiores del Alpino en las altas canales del interior de la peña (Conopodium majus majus/Jasione laevis) con registros medios entre 6,5 y $5,6^{\circ} \mathrm{C}$ y mínimos absolutos de $-18^{\circ} \mathrm{C}$.

En el caso de las bioclimáticas se diferencian dos agrupaciones. Los melojares arborescentes y los matorrales de sustitución (Trifolium pratense pratense var pratense/Vaccinium myrtillus) desde el Altimontano superior al Subalpino inferior (16.6) que se localizan en la solana meridional del macizo forman la primera agrupación (57). En la segunda (44) se incluyen las formaciones supraforestales del Subalpino inferior y el Alpino inferior (Festuca eskia/Silene nutans subsp. nutans) comprendidas entre los niveles superiores.

La agrupación mejor representada en las variables morfofisiográficas (35) se asocia a las vertientes subalpinas y alpinas petranas sobre conglomerados, tanto en orientación norte como sur. Incluye enclaves húmedos colonizados por pastos con un cierto grado de nitrificación que coinciden con sesteaderos o pastaderos de ganado. Como especies características destacan Trifolium pratense pratense var pratense y Aquilegia vulgaris subsp. vulgaris. Un segundo grupo (33) coloniza crestas y collados altos dominados por especies que fijan las inestables y acentuadas vertientes de las canales superiores, así como los pedregales sobre conglomerados del Altimontano y Subalpino inferior (Saxifraga stellaris/Deschampsia flexuosa). Los roquedales térmicos y piedemonte pizarroso meridional en el Altimontano sometidos a activas dinámicas solifluidales (Genista obtusiramea/Pimpinella major) constituyen la tercera agrupación (33).

En el caso de la insolación el grupo mejor representado (49) es el de los pastizales de cumbres, crestas, collados altos, matorrales de vertientes altas y formaciones rupícolas desde el Subalpino superior al Alpino (medias de 8.1 y $8.5 \mathrm{~h}$ ) con Poa bulbosa/Agrostis capillaris/Agrostis delicatula/Agrostis rupestris). Un segundo grupo (30) presenta medias de $6.9 \mathrm{~h}$ y se asocia a las formaciones higrófilas y matorrales subarbustivos del piedemonte rocoso septentrional. En el último conjunto (12) se agrupan pastos y humedales subalpinos (Saxifraga stellaris/Rumex acetosella angiocarpus) con valores de $5.6 \mathrm{~h}$ y 9 y matorrales arbustivos y robledales (Genista obtusiramea/Minuartia recurva) del Altimontano al Subalpino inferior $(9.3 \mathrm{~h})$. 


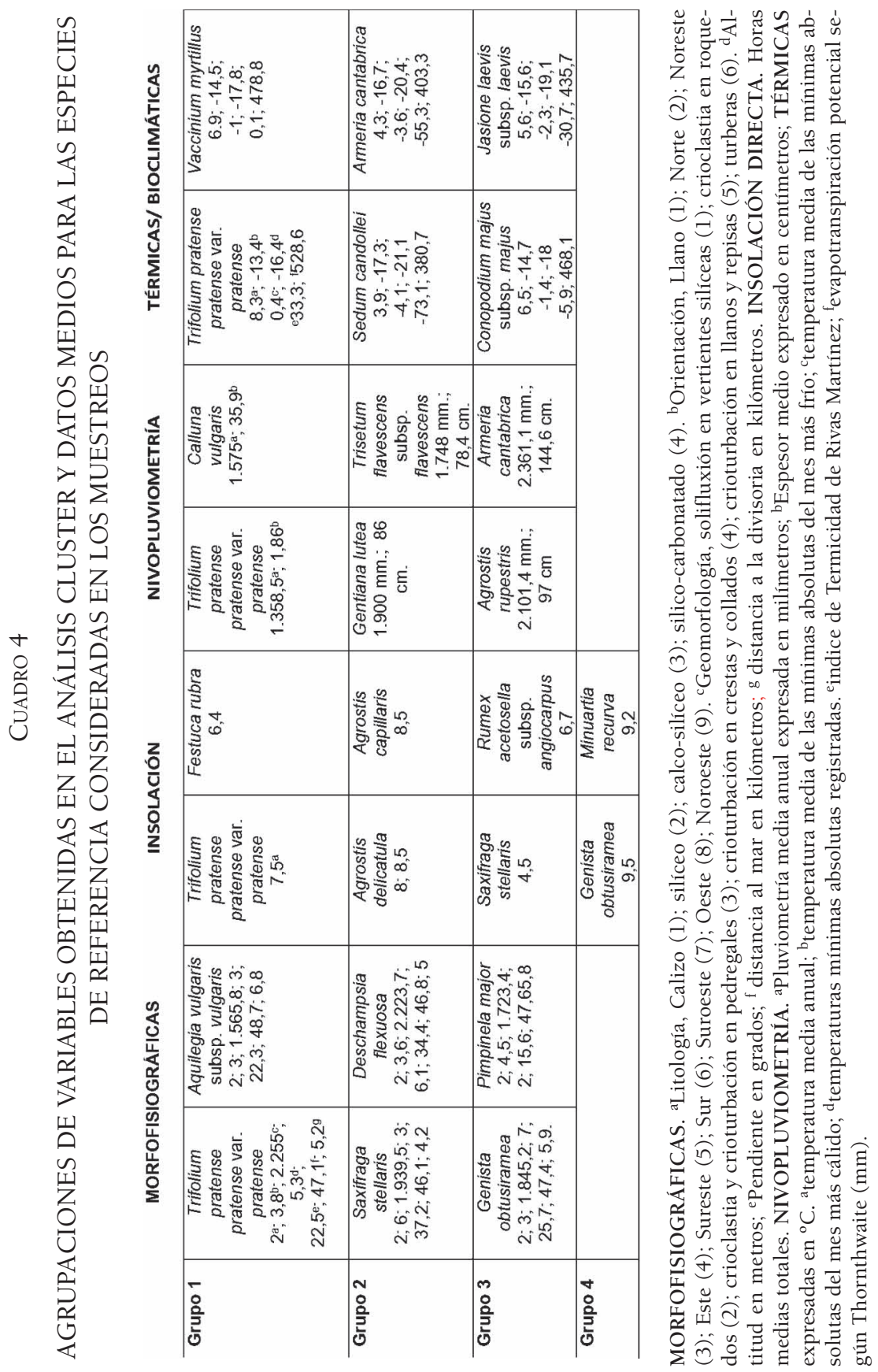

Estudios Geográficos, Vol. LXXVI, 278, pp. 7-38, enero-junio 2015

ISSN: 0014-1496, eISSN: 1988-8546, doi: 10.3989/estgeogr.201501 
En último lugar y dentro de las variables nivopluviométricas se diferencia un primer grupo con los valores de espesor nival y precipitación más bajos (51) y engloba las comunidades vegetales altimontanas y del Subalpino inferior incluyendo abedulares, robledales, genistedos y comunidades heliófilas de arroyos (Trifolium pratense pratense var. pratense/Calluna vulgaris). Los pedregales y canales del Subalpino superior y Alpino inferior forman el segundo (29) con incremento general de los valores altitudinales, pluviométricos y de espesor medio de nieve (Gentiana lutea/Trisetum flavescens flavescens). El último grupo considera los pastos y matorrales subarbustivos petranos alpinos (Agrostis rupestris/Armeria cantabrica) donde se alcanzan los registros más elevados (21).

\section{CONCLUSIONES}

En la información obtenida de la modelización de los datos climáticos resultan determinantes las relaciones entre variables. Las térmicas y bioclimáticas están íntimamente relacionadas debido al peso alcanzado por las primeras en el cálculo de las segundas. En este caso al considerarse en el cálculo una cantidad importante de variables se obtienen información de mayor de detalle. Las morfofisiográficas están condicionadas por la masividad del afloramiento conglomerático, la extensa superficie cubierta por canturrales conglomeráticos y por la contundencia morfológica de los materiales del piedemonte pizarroso. Todos estos elementos, unidos a una acusada pendiente, resultan definitivos en la existencia de una flora condicionada por la alternancia de pequeños rellanos donde domina la crioturbación o por una elevada inestabilidad superficial donde la solifluxión o las pedreras son frecuentes. En el caso de la altitud y, debido a la condicionalidad impuesta sus elevados valores medios de partida, su influencia es menor. La orientación adquiere cierto peso en la definición del conjunto al perfilar con cierto detalle la sutileza de las sombras orográficas peor definidas. En cuanto a la insolación existe una mayor dispersión en las agrupaciones asociada a la complicada articulación interna del propio macizo. La nivopluviometría, debido a la heterogeneidad y escasez de datos y a las escasas variables consideradas en su análisis, adquiere una relevancia menor que en los casos anteriores.

De la interpretación de los parámetros climáticos y de la catalogación y análisis de la vegetación consideramos que el Curavacas se puede caracterizar como una montaña esencialmente eurosiberiana suboceánica. A los datos climáticos se suma la filiación corológica de las especies muestreadas que en un 
43,3\% son eurosiberianas y en un 9,9\% atlánticas. No obstante, el detalle de nuestro análisis nos permite matizar esta apreciación a escala de vertientes. La vertiente norte podríamos calificarla de oceánica, frente a la Sur y algunos sectores de la septentrional, donde los valores térmicos y la disminución de la pluviometría introducen rasgos típicamente continentales. De ahí que en estos sectores podríamos calificar esta montaña como submediterránea. De estas matizaciones se exceptúan los pastizales psicroxerófilos de cumbres, crestas y umbrías próximas a la culminación donde a los rigores propios del Subalpino superior y de los niveles inferiores del Alpino se suman rasgos propios de un clima de alta montaña reforzados por la presencia de taxones de origen boreoalpino y circumboreal.

Fecha de recepción: 3 de enero de 2014.

Fecha de aceptación: 3 de diciembre de 2014.

\section{BiBLIOGRAFíA}

Allende Álvarez, F. (2006): "Las comunidades vegetales sobre calizas del macizo del Espigüete (Palencia-León)”. Serie Geográfica, 13, pp. 81-97.

Allende Álvarez, F. (2007): "Clima y vegetación del sector centro-oriental de la cordillera Cantábrica". Tesis doctoral. Universidad Autónoma de Madrid.

Allende Álvarez, F. (2008): "Una aproximación a la caracterización climática de un sector de la montaña central cantábrica". Estudios Geográficos, 69, pp. 357-383.

Bedia, J.; Busqué, J. y Gutiérrez, J. M. (2011): "Predicting plant species distribution across an alpine rangeland in northern Spain. A comparison of probabilistic methods". Applied Vegetation Science, 14, pp. 415-432.

Braun-Blanquet, J. (1979): Fitosociología. Bases para el estudio de las comunidades vegetales. Madrid, Blume ediciones.

Escudero, A.; Gavilán, R. y Rubio, A. (1984): "Una breve revisión de técnicas de análisis multivariantes aplicables en Fitosociología". Botanica Complutense, 19, pp. 9-38 p.

Felicísimo Pérez. A. M. (1994): Modelos digitales del terreno. Introducción y aplicación en las ciencias ambientales. Oviedo, Pentalfa ediciones.

Felicísimo, A. M. (coord.) (2011): Impactos, vulnerabilidad y adaptación al cambio climático de la biodiversidad española. 2. Flora y vegetación. Madrid, Oficina Española de Cambio Climático, Ministerio de Medio Ambiente y Medio Rural y Marino.

Fernández García, F. (1981): "La disimetría pluviométrica entre las vertientes norte y sur del Sistema Central”, VII Coloquio de Geografía, Pamplona, pp. 91-97.

Fernández García, F; Soriano Carrillo, J.; Allende Álvarez, F. y Galán Gallego, E. 1996: "Delimitación espacial de la precipitación útil en la Comunidad de Madrid me- 
diante la utilización de un Sistema de Información Geográfica", en P. Valladares, (coord.): Clima y agua. La gestión de un recurso climático. Universidad de la Laguna, pp. 311-323.

Fotheringham, S. y Rogerson, P. (eds.) (1994): Spatial Analysis and GIS. Buffalo, Taylor Francis, 281 pp.

Frochoso, M. y Castañón, J. C. (1996): "El relieve heredado de la glaciación cuaternaria en el Macizo de Peña Prieta (Cordillera Cantábrica)". Polígonos, 6, pp. 25-43.

García Fernández, J. (1986): El Clima en Castilla y León, León, Ámbito.

Green, D. R. y Cousins, S. H. (eds.) (1993): Landscape Ecology and GIS. Londres, Taylor Francis, 288 pp.

Herrero Cembranos, L. (1986): Estudio de los pastizales psicroxerófilos silicícolas de la provincia de León. Celarayn, León, Institución Fray Bernardino de Sahagún.

Hutchinson, M. F. (1993): "Development of a continent-wide DEM with applications to terrain and climate analysis", en M. F. Goodchild et al. (eds.): Environmental Modeling with GIS. New York, Oxford University Press, pp. 392-399.

Loriente Escallada, E. (1991): "Una aproximación al conocimiento de la vegetación y flora del piso subalpino superior de la Peña Prieta de Cantabria". Anales del Instituto de Estudios Agropecuarios, 12, pp. 151-166.

Martín Vide, J. y Olcina Cantos, J. (2001): Clima y tiempos de España. Historia y Geografía. Madrid, Alianza Editorial.

Ministerio de Obras Públicas, Transportes y Medio Ambiente. Programa EHRIN (1994a): La nieve en las cordilleras españolas. Año 1991-92. Madrid, MOPTMA.

Ministerio de Obras Públicas, Transportes y Medio Ambiente. Programa EHRIN (1994b): la nieve en las cordilleras españolas. Año 1993-94. Madrid, MOPTMA.

Ministerio de Obras Públicas, Transportes y Medio Ambiente. Programa EHRIN (1995a): La nieve en las cordilleras españolas. Año 1992-93. Madrid, MOPTMA.

Ministerio de Obras Públicas, Transportes y Medio Ambiente. Programa EHRIN (1995b): la nieve en las cordilleras españolas. Año 1994-95. Madrid, MOPTMA.

Nava, H. S. (1988): "Flora y vegetación orófila de los Picos de Europa". Ruizia, 6.

Pauli, H.; Gottfried, M.; Hohenwallner, D.; Reiter, K. y Grabherr, G. (2003): Manual para el trabajo de campo del proyecto GLORIA. Luis Villar, Versión española. Zaragoza, Sdad. Coop. de Artes Gráficas, 63 pp. + Anexos.

Pellitero, R. (2013a). "Evolución finicuaternaria del glaciarismo en el macizo de Fuentes Carrionas (Cordillera Cantábrica), propuesta cronológica y paleoambiental". Cuaternario y Geomorfología, 27 (1-2), 71-90.

Pellitero, R. (2013b): "Geomorphology and geomorphological landscapes of Fuentes Carrionas", Journal of Maps, doi: 10.1080/17445647.2013.867822. http://dx.doi. org/10.1080/17445647.2013.867822 (Verificada el 29/4/2015)

Podani, J. (1984): "Analysis of mapped and simulated vegetation patterns by means of computerized sampling techniques". Acta Bot. Acad. Sci. Hung, 30, pp.403-425

Price, F. y Heywood, D. (eds.) (1994): Mountain Environments E Geographic Information Systems. Londres, Taylor Francis, 220 pp. 
Rivas-Martínez, S.; Díaz, T. E.; Fernández Prieto, J. A.; Loidi. J. y Penas, A. (1984): la vegetación de la alta montaña cantábrica: los Picos de Europa. León, Ediciones Leonesas.

Rivas-Martínez, S. (2008): Globalbioclimatics. http://www.globalbioclimatics.org [Consulta: noviembre 2011. Verificada: 29/4/2015].

Rodríguez Fernández, L. R. (1994): La estratigrafía del paleozoico y la estructura de la región de Fuentes Carrionas y áreas adyacentes (Cordillera herciniana, NO España). O Castro, Laboratório Xeologico de Laxe, 240 pp. Serie Nova Terra.

SIVIM. Sistema de Información de la Vegetación Ibérica y Macaronésica http://www.sivim.info/sivi/ [Consulta cuadrícula 30TUN56: noviembre 2011 Verificada: 29/4/2015].

Tuhkanen, S. (1980): "Climatic parameters and indices in plant geography". Acta Phytogeogr, 67, pp. 1-110.

\section{RESUMEN}

Se plantea una caracterización fitoclimática de un macizo montañoso incluido en la montaña central cantábrica y culminado por el pico Curavacas $(2.524 \mathrm{~m})$. Como punto de partida se utilizan muestreos de campo de vegetación en combinación con los datos térmicos y pluviométricos normalizados (39 y 38 estaciones respectivamente) obtenidos del SIGA (Servicio de Información Geográfica de Datos Agrarios) y los de 25 pértigas de medición de espesor nival (Programa EHRIN). La información se trató mediante técnicas estadísticas de análisis multivariado y de análisis cluster con el fin de modelizar espacialmente los resultados mediante un sistema de información geográfica. En el trabajo se analizan las diferentes variables que intervienen, su validez y su relación con los elementos que resultan determinantes en la explicación de la distribución de la vegetación: altitud, latitud, longitud, orientación, pendiente o distancia a la divisoria de aguas y al mar. Los resultados obtenidos reflejan la fortaleza de las variables térmicas, bioclimáticas y geomorfológicas como predictoras de la distribución de la vegetación. Lo anterior, unido a la filiación corológica de los taxones, permite caracterizar y clasificar el comportamiento fitoclimático de esta montaña y considerarla como de tipo eurosiberiano suboceánico.

PAlabras Clave: cordillera Cantábrica; clima de montaña, clasificación fitoclimática; sistemas de información geográfica; estadística multivariada; vegetación de alta montaña.

\section{AbSTRACT}

This article deals a phytoclimatic characterization of a mountain massif included in Cantabrian central mountain and dominated by Curavacas peak $(2.524 \mathrm{~m})$. As starting point are utilized field sampling vegetation plots combining with rainfall and thermal normalized data obtained of the SIGA (Service of Agrarian Geographical 
Information) and the data of 25 poles of measurement snow depth (EHRIN program). The data was processed by statistical techniques (multivariate and Cluster analysis) for modelling the results with a geographical information system. The paper analyse the different abiotic variables (altitude, latitude, longitude, aspect, slope or distance to the watershed or to the sea) that influence in the distribution of vegetation and its relationships with climatic factors: temperature, rainfall and snow depth. The results reflect the strengths of bioclimatic, temperature and geomorphologic variables as predicting of the distribution of vegetation. In addition, corological areas of identified taxa make it possible the classification and characterization of this massif as a eurosiberian-suboceanic type.

KEY WORDS: Cantabrian mountain range; mountain climate; phytoclimatic classification; geographical information systems; multivariate statistics; high-mountain vegetation.

\section{RÉSUMÉ}

On aborde la caractérisation phyto-climatique d'un ensemble de massifs inclus dans la montagne centrale cantabrique et dominée par Le Pic du Curavacas (2.524 m). Comme point de départ on utilise techniques d échantillonnage de la végétation en combinaison avec les données thermiques et pluviométriques régularisées (39 et 38 stations météo respectivement) obtenues du SIGA (Service d'Information Géographique Agricole) et ceux de 25 perches de mesure d'épaisseur nivaux (Programme EHRIN). Linformation est traité au moyen de techniques statistiques d'analyse multivarié et d'analyse cluster, afin de modéliser les différentes variables qui participent, sa validité et sa relation avec des éléments qui sont déterminants dans leur explication la répartition de la végétation: altitude, latitude, longitude, orientation, pente ou distance à la divisoire des eaux et à la mer. Les résultats reflètent la force des variables thermiques et bioclimatiques comme facteurs prédictifs de la répartition de la végétation. Ceci, combiné avec des détails de modélisation de surface et l'affiliation chorologique de taxons nous permet de caractériser et classer le comportement phytoclimatique de cette montagne et considérons le type sub-océaniques comme Eurosiberian.

Mots CLÉS: chaîne cantabrique; climat de montagne; classification phyto-climatique; systèmes d'information géographique ; régression multiple; végétation de haute montagne. 


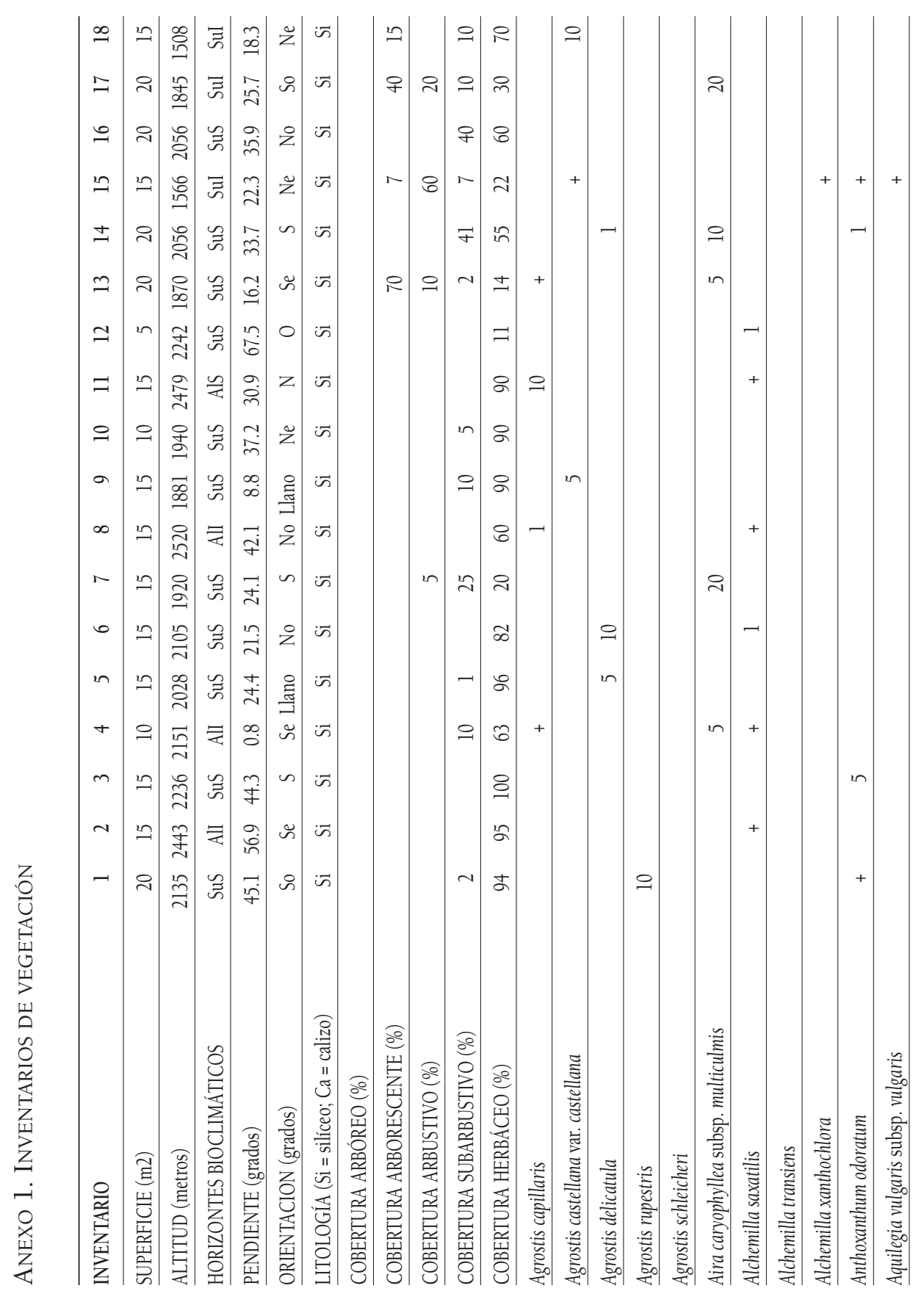

Estudios Geográficos, Vol. LXXVI, 278, pp. 7-38, enero-junio 2015

ISSN: 0014-1496, eISSN: 1988-8546, doi: 10.3989/estgeogr.201501 


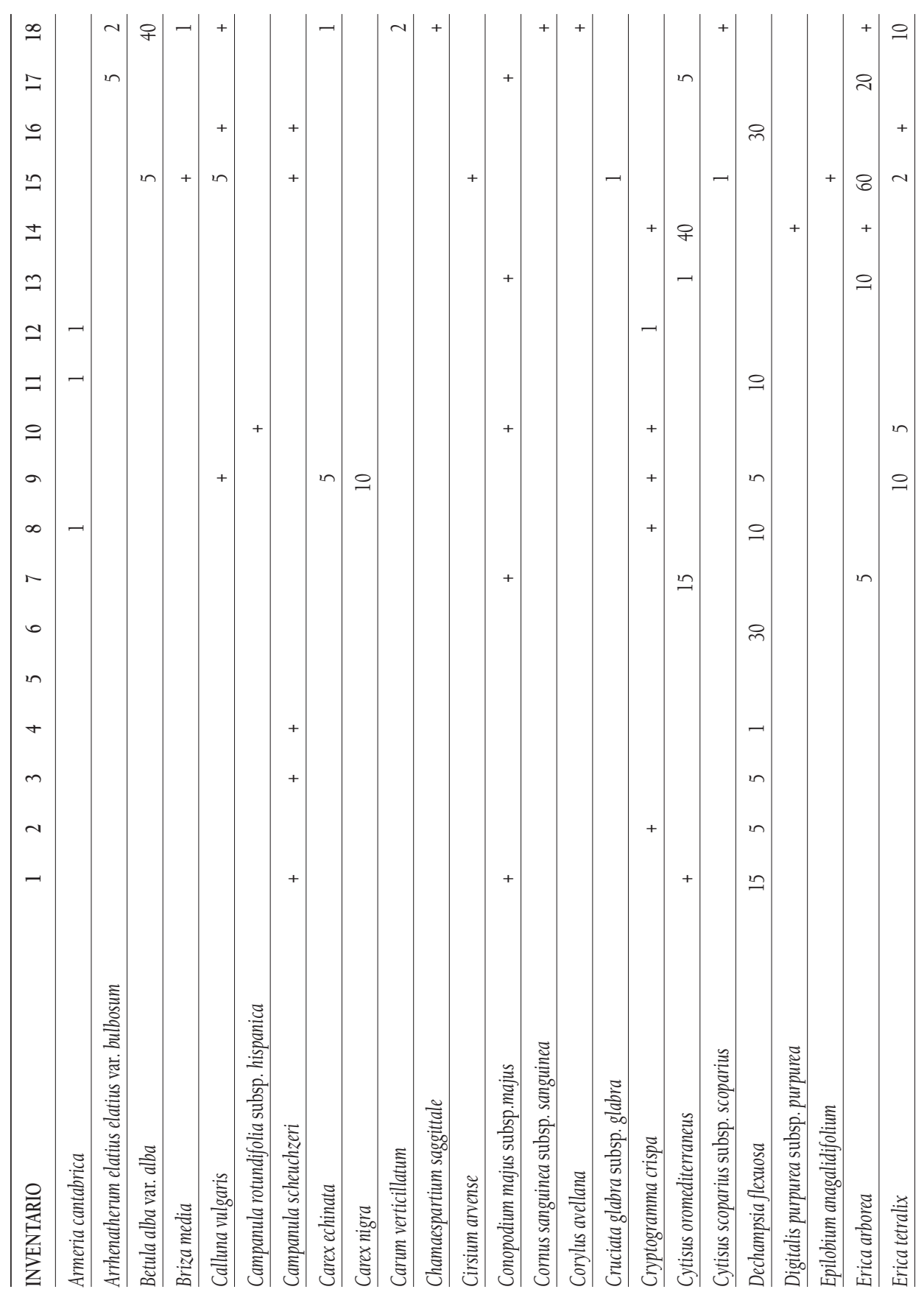

Estudios Geográficos, Vol. LXXVI, 278, pp. 7-38, enero-junio 2015 ISSN: 0014-1496, eISSN: 1988-8546, doi: 10.3989/estgeogr.201501 


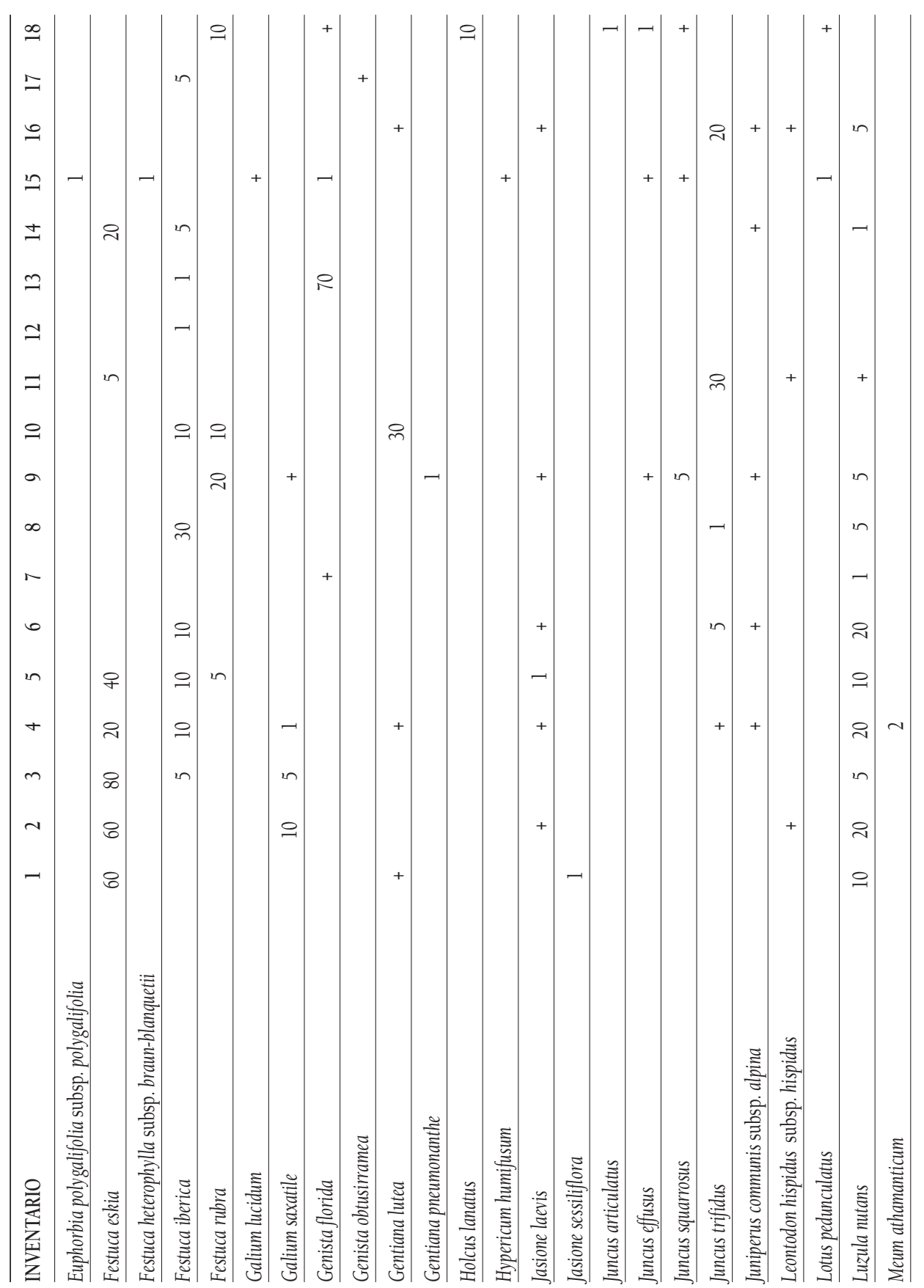

Estudios Geográficos, Vol. LXXVI, 278, pp. 7-38, enero-junio 2015

ISSN: 0014-1496, eISSN: 1988-8546, doi: 10.3989/estgeogr.201501 


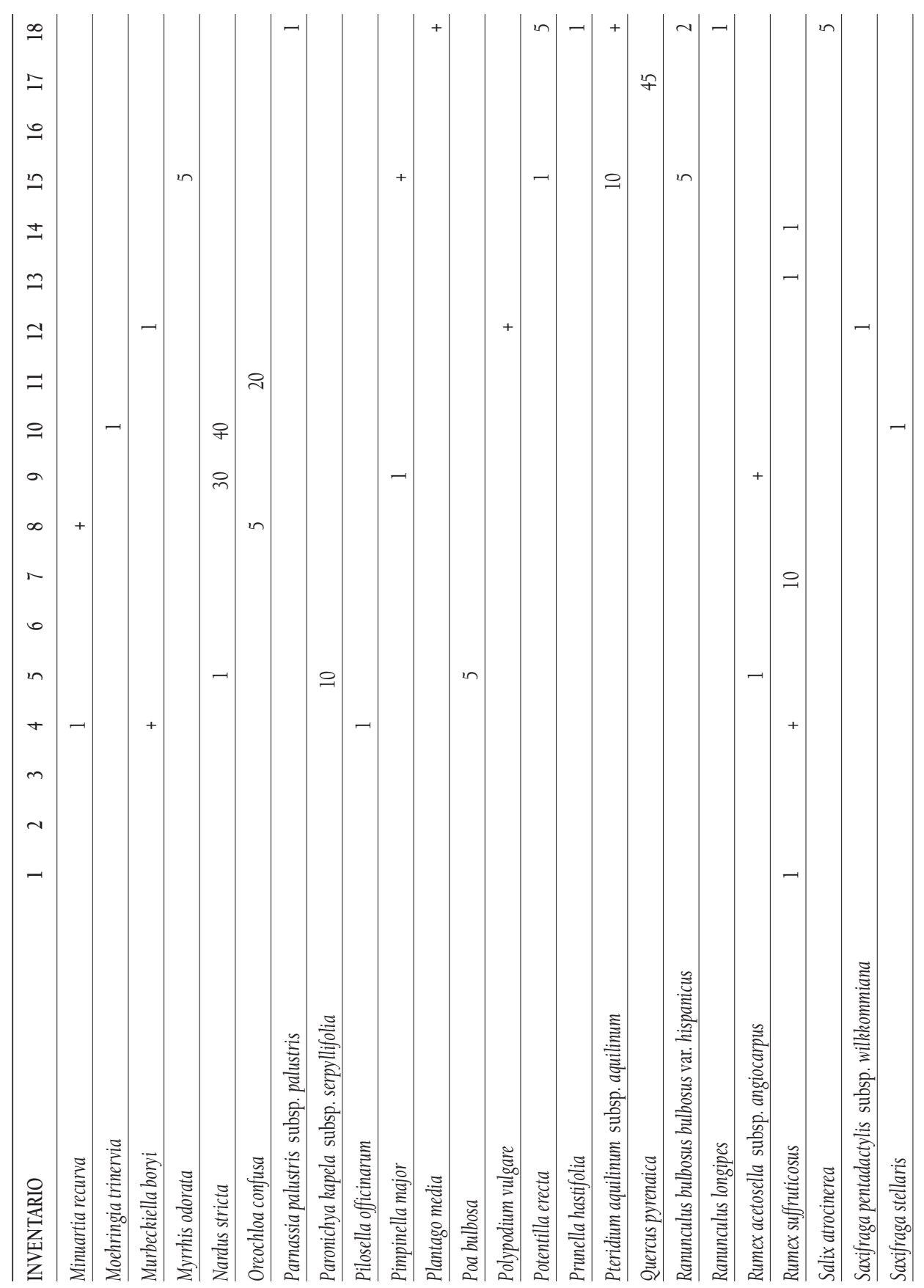

Estudios Geográficos, Vol. LXXVI, 278, pp. 7-38, enero-junio 2015 ISSN: 0014-1496, eISSN: 1988-8546, doi: 10.3989/estgeogr.201501 


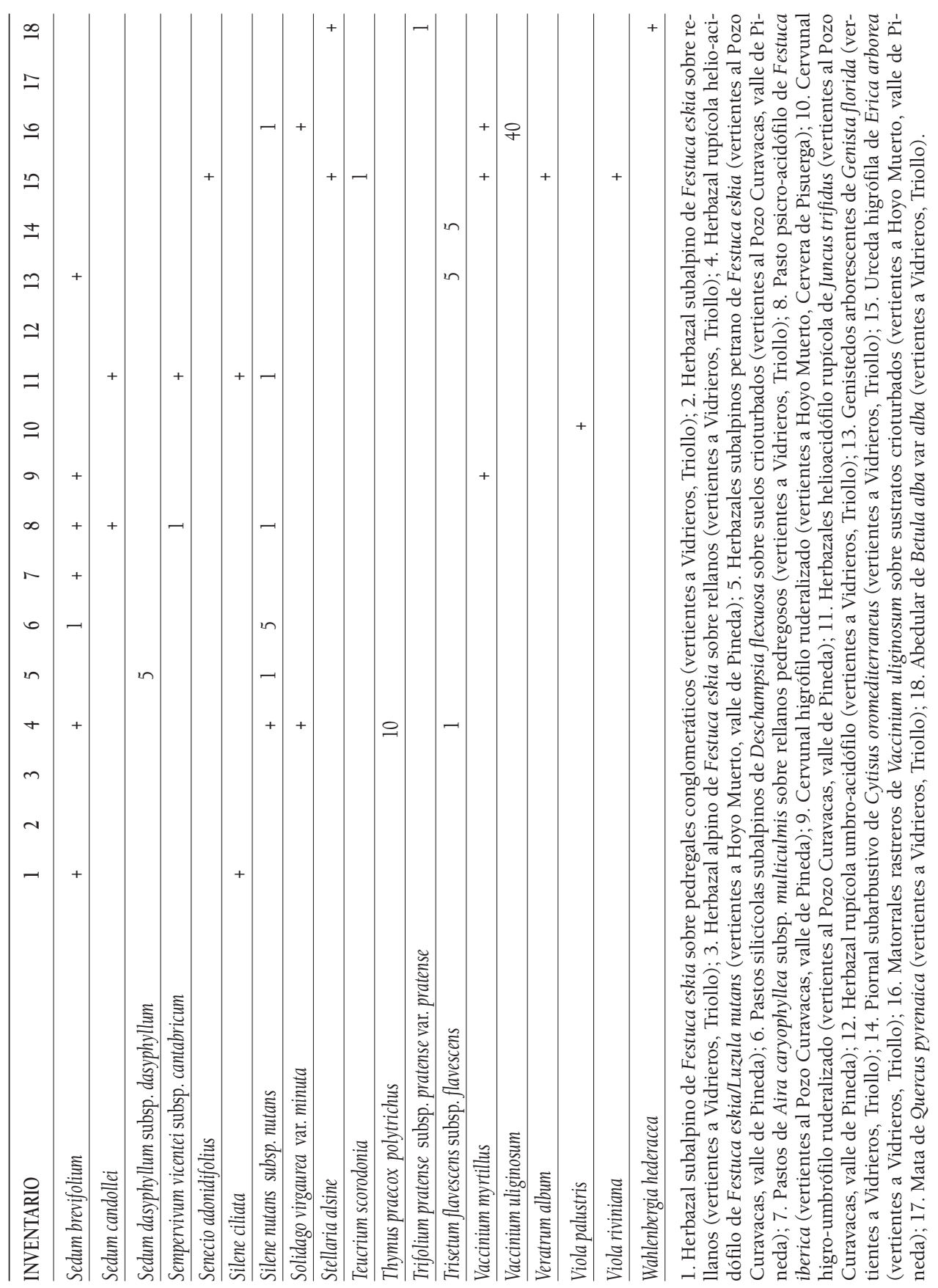

Estudios Geográficos, Vol. LXXVI, 278, pp. 7-38, enero-junio 2015

ISSN: 0014-1496, eISSN: 1988-8546, doi: 10.3989/estgeogr.201501 